\title{
Suppression of decoherence by bath ordering
}

\author{
Jun Jing *, H.R. Ma \\ Institute of Theoretical Physics, Shanghai Jiao Tong University \\ 800 DongChuan Road, MinHang, Shanghai 200240, China
}

(Dated: December 8, 2018)

\begin{abstract}
The dynamics of two coupled spins- $1 / 2$ coupled to a spin-bath is studied as an extended model of the Tessieri-Wilkie Hamiltonian [1]. The pair of spins served as an open subsystem were prepared in one of the Bell states and the bath consisted of some spins- $1 / 2$ is in a thermal equilibrium state from the very beginning. It is found that with the increasing the coupling strength of the bath spins, the bath forms a resonant antiferromagnetic order. The polarization correlation between the two spins of the subsystem and the concurrence are recovered in some extent to the isolated subsystem. This suppression of the subsystem decoherence may be used to control the quantum devices in practical applications.

PACS numbers: 03.65.-w, 03.67.-a, 05.30.-d
\end{abstract}

\footnotetext{
* Email: jingjun@sjtu.edu.cn
} 


\section{INTRODUCTION}

Quantum decoherence is a common and inevitable phenomenon in an open quantum system due to its interaction with the surrounding bath or environment. An initial superposition state of the system, $\rho_{s}(t=0)=\left|\psi_{0}\right\rangle\left\langle\psi_{0}\right|$, has to decay into a classical mixture of states, $\rho_{s}=\sum_{i} p_{i} \rho_{i}, \sum_{i} p_{i}=1$, after a decoherence time $\tau_{d}$. During the same period of time, some of the information in the initial state of the system might irreversibly lose into the bath [2, 3, 4, 5, 6]. A real-world system, for instance a quantum device or qubit, cannot be completely isolated from the environment. In the last two decades, there are great interests in the search for realizations of quantum computation as well as quantum communications, such technologies rely on the possibilities that quantum devices can be manufactured with negligible decoherence in the period of operation. Since decoherence is intrinsic to open quantum systems, the problem is transformed to a problem on how to reduce or eliminate the decoherence of an open quantum system. Many works have been devoted into the research of the influences caused by the subsystem-bath coupling and the decoherence dynamics of open quantum systems [7, 8], for the possible realizations of quantum communication and quantum computation.

A very important part of any theoretical research of decoherence is the modeling of the bath or environment. There had been mainly two important types of bath schemes: (i) the bath consisted of harmonic oscillators, e.g., the Caldeira-Leggett model [9]; (ii) the bath made up of spins-1/2, e.g., model used in Ref. [10]. For both types of bath model, there are typically two kinds of approximations, Markovian [11] or non-Markovian [12], used in the studies. The focus of most of the researches were on the eliminating the destructive effects of the environment to the system. However, Tessieri and Wilkie proposed a new idea to the reducing of decoherence [1] of the open subsystem, which is a spin-1/2 coupled to a low-temperature bath of spins. They introduced the coupling terms between bath spins into their spin-bath Hamiltonian, which is a simplification of the vibronic Hamiltonian of the impurity and crystal [13] using spin-1/2 modes. One of the most important results they found is that the strong antiferromagnetic intra-bath interactions could be utilized to make the dynamics of the central spin almost autonomous from the bath around. And Dawson et al. [14] also found the decoherence effect 
of the bath can be suppressed by the increasing intra-bath coupling in Tessieri-Wilkie model.

Inspired by their works and the papers of Xiang et al. [15] and Liu et al. [16], the aim of this paper is to study the dynamics of correlation characteristics between the two coupled center spins, which constitute our subsystem. The bath in our study is the same model used by the Ref. [1, 14], which is prepared in a thermal equilibrium state at time $t=0$. The dynamics of the subsystem and the bath then calculated, phenomena such as decoherence oscillation were observed, which can be quantified by the amplitudes of polarization components $\left\langle\sigma_{x}\right\rangle,\left\langle\sigma_{y}\right\rangle$ and $\left\langle\sigma_{z}\right\rangle$. We studied the evolution of the spatial correlation between polarizations of the two subsystem spins along three directions $\vec{x}, \vec{y}$ and $\vec{z}$. we also discussed the entanglement between the two system spins [17], which is an essential ingredient in the quantum communication [18, 19] and has no classical counterpart. The concurrence of the subsystem was chosen to be a measure of the entanglement between the two spins within the subsystem [20, 21]. It will be demonstrated that in our extended model, both the polarization correlation and entanglement of the subsystem can be restored to a great extent to the isolated case by the increasing pairwise couplings between the bath spins. And we also try to clarify the physics behind this revival function of intra-bath coupling. The rest of this paper is organized as follows. In section III we introduce the Hamiltonian for our two-center-spins-spin-bath model; In section III, we explain the computation procedures about the evolution of the correlation and of the concurrence for the subsystem; Detailed results and discussions are in section IV. The conclusion of our study is given in section V.

\section{THE EXTENDED SPIN-SPIN-BATH MODEL}

The subsystem we studied here consists of two spins antiferromagneticly coupled in the $x$ direction, and aligned in $x$ and $z$ direction respectively by applied fields. The bath consists of $N-2$ spins, every pair of the spins are also coupled antiferromagneticly in the $x$ direction, and aligned in both $x$ and $z$ direction. The subsystem interacts with the bath by the antiferromagnetic coupling in the $x$ direction. This model is an extension to the Tessieri-Wilkie model [1], which comes from the simplification of an experimentally realizable model system. The original model is based on the system that an interstitial He atom in an otherwise pure silicon or diamond cluster, the He atom causing small lattice 
distortion in the cluster [22], thus inducing vibronic coupling between the electronic states and phonon states of the cluster. This mechanism is further simplified by considering only the ground state and the first excited state of the interstitial He atom, representing the states with a spin-1/2 Pauli matrix, and representing the phonon states as spin-1/2 modes, which resulting in the Tessieri-Wilkie model. This model system is regarded as a potential realization for quantum computing device. Our extension is that instead of one center spin, we have two center spins coupled together by an antiferromagnetic coupling. In this extension we merely regard the Tessieri-Wilkie model as a model system and did not go into details of the physical realization. So that we will only focus our attention to the extended model itself in the following discussions.

The Hamiltonian of our system can be written as:

$$
\begin{aligned}
H & =H_{S}+H_{B}+H_{S B} \\
H_{S} & =\frac{\omega_{s}}{2} \sigma_{z}^{(1)}+\beta \sigma_{x}^{(1)}+\frac{\omega_{s}}{2} \sigma_{z}^{(2)}+\beta \sigma_{x}^{(2)}+\lambda_{s s} \sigma_{x}^{(1)} \sigma_{x}^{(2)}, \\
H_{B} & =\sum_{i=3}^{N} \frac{\omega_{b}}{2} \sigma_{z}^{(i)}+\beta \sum_{i=3}^{N} \sigma_{x}^{(i)}+\lambda_{b b} \sum_{i=3}^{N-1} \sum_{j=i+1}^{N} \sigma_{x}^{(i)} \sigma_{x}^{(j)}, \\
H_{S B} & =\lambda_{s b} \sum_{i=3}^{N}\left(\sigma_{x}^{(1)} \sigma_{x}^{(i)}+\sigma_{x}^{(2)} \sigma_{x}^{(i)}\right) .
\end{aligned}
$$

Where $H_{S}$ is the Hamiltonian of the subsystem with two interacting spins (labeled by 1,2 ) coupled in $x$ direction with coupling strength $\lambda_{s s} . \beta, \omega_{s} / 2$ and $\omega_{b}$ may be regarded as applied fields acting in $x$ and $z$ directions to the sub-system and bath spins. The $\sigma_{x}$ and $\sigma_{z}$ are the Pauli matrices:

$$
\sigma_{x}=\left(\begin{array}{ll}
0 & 1 \\
1 & 0
\end{array}\right), \quad \sigma_{z}=\left(\begin{array}{cc}
1 & 0 \\
0 & -1
\end{array}\right)
$$

The bath consists of $N-2$ spins labeled by 3 to $N$, coupled each other in the $x$ direction with coupling strength $\lambda_{b b}$, denoted as $H_{B}$. The coupling between subsystem and bath is given by $H_{S B}$, which is also the coupling of each subsystem spin with every bath spin in $x$ direction, with coupling strength $\lambda_{s b}$. All the interactions are antiferromagnetic, that is

$$
\lambda_{s s} \geq 0, \quad \lambda_{b b} \geq 0, \quad \lambda_{s b} \geq 0
$$

In the following calculation, we use the system of units where the Boltzmann constant $k_{B}=1$ and Plank constant $\hbar=1$. The other parameters in the Hamiltonian take the 
following values in the subsequent computations:

$$
\omega_{s}=0.8, \quad \beta=0.1, \quad \omega_{b}=1.0
$$

\section{CALCULATION PROCEDURES}

In this section, we describe in detail the algorithms for the calculation of the dynamics of the subsystem modeled by the Hamiltonian (11). The initial state of the subsystem is chosen to be one of the four Bell states:

$$
\begin{aligned}
& \left|\psi_{S}(0)\right\rangle^{1}=1 / \sqrt{2}(|11\rangle+|00\rangle), \\
& \left|\psi_{S}(0)\right\rangle^{2}=1 / \sqrt{2}(|10\rangle+|01\rangle), \\
& \left|\psi_{S}(0)\right\rangle^{3}=1 / \sqrt{2}(|11\rangle-|00\rangle), \\
& \left|\psi_{S}(0)\right\rangle^{4}=1 / \sqrt{2}(|10\rangle-|01\rangle) .
\end{aligned}
$$

where $|0\rangle$ and $|1\rangle$ refer to the spin "down" and spin "up" in the $z$ direction, respectively. The reason that we use these states as initial states is that among all the quantum states for a pair of coupled spin-1/2, Bell bases have the largest degree of entanglement and many other interesting characteristics [23]. However, it should be noted that the last Bell state given in equation (10) is a state free from the bath, the action of the interaction part of the Hamiltonian, $H_{S B}$, on the state gives zero. So that the state will evolve with time just as an isolated system. Further more, the state is also an eigenstate of the subsystem Hamiltonian $H_{s}$ :

$$
H_{s}(1 / \sqrt{2}(|10\rangle-|01\rangle))=-1 / \sqrt{2} \lambda_{s s}(|10\rangle-|01\rangle) .
$$

The time evolution of the state is simply an exponential factor $e^{i \omega t}$, with $\omega=-\lambda_{s s}$. So that the physical quantities will not vary with time in this state and we will not consider it in the following calculations. The initial state of the bath is taken to be the thermal equilibrium state: $\rho_{B}(0)=Z^{-1} \exp \left(-H_{B} / T\right)$, where $Z$ is the partition function of the environment $Z=\operatorname{Tr}\left(\exp \left(-H_{B} / k_{B} T\right)\right)$. The density matrix $\rho(t)$ of the whole system can formally be represented as:

$$
\begin{aligned}
\rho(t) & =\exp (-i H t) \rho(0) \exp (i H t) \\
\rho(0) & =\rho_{S}(0) \otimes \rho_{B}(0) \\
\rho_{S}(0) & =\left|\psi_{S}(0)\right\rangle\left\langle\psi_{S}(0)\right|
\end{aligned}
$$


In order to find the density matrix $\rho(t)$, we follow the method suggested by Tessieri et al 1]. The thermal bath state $\rho_{B}(0)$ can be expanded with the eigenstates of the environment Hamiltonian:

$$
\begin{aligned}
\rho_{B}(0) & =\sum_{n=1}^{2^{m}}\left|\phi_{n}^{(B)}\right\rangle \omega_{n}\left\langle\phi_{n}^{(B)}\right|, \\
\omega_{n} & =\frac{e^{-E_{n} / T}}{Z}, \\
Z & =\sum_{n=1}^{2^{m}} e^{-E_{n} / T} .
\end{aligned}
$$

Here $\left|\phi_{n}^{(B)}\right\rangle, n=1,2,3, \cdots, 2^{m}, m=N-2$, are the eigenstates of the environment Hamiltonian $H_{B}$, and $E_{n}$ the corresponding eigen energies. With this expansion, the density matrix $\rho(t)$ can be written as:

$$
\rho(t)=\sum_{n=1}^{2^{m}} \omega_{n}\left|\Psi_{n}(t)\right\rangle\left\langle\Psi_{n}(t)\right| .
$$

Where

$$
\left|\Psi_{n}(t)\right\rangle=\exp (-i H t)\left|\Psi_{n}(0)\right\rangle=U(t)\left|\Psi_{n}(0)\right\rangle
$$

The initial state is

$$
\left|\Psi_{n}(0)\right\rangle=\left|\psi_{S}(0)\right\rangle\left|\phi_{n}^{(B)}\right\rangle
$$

The evolution operator $U(t)$ can be evaluated by the efficient algorithm of polynomial schemes [24, 25, 26]. The method used in this calculation is the Laguerre polynomial expansion method we proposed in Ref. [26], which is pretty well suited to this problem and can give accurate result in a comparatively smaller computation load. More precisely, the evolution operator $U(t)$ is expanded in terms of the Laguerre polynomial of the Hamiltonian as:

$$
\begin{aligned}
U(t) & =e^{-i H t} \\
& =\left(\frac{1}{1+i t}\right)^{\alpha+1} \sum_{k=0}^{\infty}\left(\frac{i t}{1+i t}\right)^{k} L_{k}^{\alpha}(H)
\end{aligned}
$$

where $\alpha$ distinguishes different types of Laguerre polynomials [27], $k$ is the order of the Laguerre polynomial. In real calculations the expansion has to be cut at some value of $k_{\max }$, which was taken to be 24 in this study. With the largest order of the expansion fixed, the time step $t$ is restricted to some value in order to get accurate results of the evolution 
TABLE I: the number of the bath states, $M$, needed in the calculation for different temperatures and thresholds of $\omega_{t h}$.

\begin{tabular}{|c|c|c|c|c|c|c|}
\hline Temperature T & $\leq 0.04$ & 0.05 & 0.08 & 0.10 & 0.15 & 0.20 \\
\hline$M\left(\omega_{n}>0.00001\right)$ & 8 & 8 & 28 & 70 & 70 & 70 \\
\hline$M\left(\omega_{n}>0.0001\right)$ & 1 & 8 & 8 & 28 & 28 & 70 \\
\hline$M\left(\omega_{n}>0.001\right)$ & 1 & 1 & 8 & 8 & 28 & 70 \\
\hline
\end{tabular}

operator. For longer times the evolution can be achieved by more steps. The action of the Laguerre polynomial of Hamiltonian to the states is calculated by recurrence relations of the Laguerre polynomial. The efficiency of this polynomial scheme [26] is about 8 times as that of the Runge-Kutta algorithm used in Ref. [1]. When the states $\left|\Psi_{n}(t)\right\rangle$ are obtained, the density matrix can be obtained by performing a summation in equation (18).

When the temperature is not very high, the weights for high energy states will be very small so that only few lowest states need to be considered. The maximum states to be included in the summation can be determined by setting a threshold of weight $\omega_{t h}$, and keep only those states whose weight $\omega_{n} \geq \omega_{t h}$. As an example, table (II) gives the number of states to be used for different temperatures and threshold. In this case the number of environment spins $m=8$, and couplings among the environment spins $\lambda_{b b}=4.0$. As can be seen from the table, the number of states needed is much less than the total number of states, $2^{8}=256$. In real calculation the up bound of the summation in equation (18) will be $M<<2^{m}$, chosen by the criteria specified for the accuracy of results. That is to say, equation (18) could be changed into the following equation:

$$
\rho(t)=\sum_{n=1}^{M} \omega_{n}\left|\Psi_{n}(t)\right\rangle\left\langle\Psi_{n}(t)\right| .
$$

After obtaining the density matrix of the whole system, the reduced density matrix is calculated by a partial trace operation to $\rho(t)$, which trace out the degrees of freedom of the environment:

$$
\rho_{S}(t)=\operatorname{Tr}_{B}(\rho(t)) .
$$

For the model of this paper, $\rho_{S}(t)$ is the density matrix of the open subsystem consists of two center spins, which can be expressed as a $4 \times 4$ matrix in the Hilbert space of the 
subsystem spanned by the orthonormal vectors $|00\rangle,|01\rangle,|10\rangle$ and $|11\rangle$.

After the reduced density matrix is obtained, any physical quantities of the subsystem can be obtained easily. In the following we concentrate on two important physical quantities of the subsystem which reflect the decoherence and entanglement degree of the subsystem state. These two quantities are the spatial polarization correlation and the concurrence.

\section{A. Polarization correlation}

The polarization of either spin in the subsystem is defined as

$$
\vec{P}^{(i)}(t)=\operatorname{Tr}\left(\rho^{(i)}(t) \vec{\sigma}^{(i)}\right), \quad i=1,2,
$$

where $\vec{\sigma}=\sigma_{x} \vec{i}+\sigma_{y} \vec{j}+\sigma_{z} \vec{k}$. To simplify the calculation, we may also trace out one of the spin degrees of freedom to obtain the $2 \times 2$ sub-reduced density matrix for each spin:

$$
\rho^{(i)}(t)=\operatorname{Tr}_{\bar{i}}\left(\rho_{S}(t)\right)=\left(\begin{array}{ll}
\rho^{(i)}(t)_{11} & \rho^{(i)}(t)_{10} \\
\rho^{(i)}(t)_{01} & \rho^{(i)}(t)_{00}
\end{array}\right)
$$

Here $\operatorname{Tr}_{i}$ means to trace out the other degrees of freedom of $i$, i.e. trace out 2 when $i=1$ and trace out 1 when $i=2$. Then the three components of $\vec{P}^{(i)}(t)$ are expressed as:

$$
\begin{aligned}
& \left\langle\sigma_{x}^{i}\right\rangle=\operatorname{Tr}\left(\rho^{(i)}(t) \sigma_{x}\right)=\rho^{(i)}(t)_{10}+\rho^{(i)}(t)_{01}, \\
& \left\langle\sigma_{y}^{i}\right\rangle=\operatorname{Tr}\left(\rho^{(i)}(t) \sigma_{y}\right)=i\left(\rho^{(i)}(t)_{10}-\rho^{(i)}(t)_{01}\right), \\
& \left\langle\sigma_{z}^{i}\right\rangle=\operatorname{Tr}\left(\rho^{(i)}(t) \sigma_{z}\right)=\rho^{(i)}(t)_{11}-\rho^{(i)}(t)_{00} .
\end{aligned}
$$

The polarization $\vec{P}^{(i)}(t)$ may be viewed as an indicator of quantum decoherence.

The correlation between the two subsystem spins can be described by the correlation functions defined bellow:

$$
\begin{aligned}
& C_{x x}=\left\langle\sigma_{x}^{(1)} \sigma_{x}^{(2)}\right\rangle-\left\langle\sigma_{x}^{(1)}\right\rangle\left\langle\sigma_{x}^{(2)}\right\rangle, \\
& C_{y y}=\left\langle\sigma_{y}^{(1)} \sigma_{y}^{(2)}\right\rangle-\left\langle\sigma_{y}^{(1)}\right\rangle\left\langle\sigma_{y}^{(2)}\right\rangle, \\
& C_{z z}=\left\langle\sigma_{z}^{(1)} \sigma_{z}^{(2)}\right\rangle-\left\langle\sigma_{z}^{(1)}\right\rangle\left\langle\sigma_{z}^{(2)}\right\rangle .
\end{aligned}
$$

where $\left\langle\sigma_{\alpha}^{(1)} \sigma_{\alpha}^{(2)}\right\rangle \equiv \operatorname{Tr}\left(\rho_{S}(t) \sigma_{\alpha}^{(1)} \sigma_{\alpha}^{(2)}\right), \alpha=x, y, z$. Since $\sigma_{x}^{2}=\sigma_{y}^{2}=\sigma_{z}^{2}=1$, so that $\left(\sigma_{\alpha}^{(1)}+\sigma_{\alpha}^{(2)}\right)^{2}=2+2 \sigma_{\alpha}^{(1)} \sigma_{\alpha}^{(2)}$, the correlation function is thus also a measurement of the fluctuations of the total spin of the system. 


\section{B. Concurrence}

The concurrence of the two spin-1/2 system is an indicator of their intra entanglement, which is defined as [20]:

$$
C=\max \left\{\lambda_{1}-\lambda_{2}-\lambda_{3}-\lambda_{4}, 0\right\}
$$

where $\lambda_{i}$ are the square roots of the eigenvalues of the product matrix $\rho_{S} \tilde{\rho}_{S}$ in decreasing order. Equation (29) applies to all kinds of states, either mixed or pure. The matrix $\tilde{\rho}_{S}$ is constructed as $\left(\sigma_{y} \otimes \sigma_{y}\right) \rho_{S}^{*}\left(\sigma_{y} \otimes \sigma_{y}\right)$. If the bipartite quantum state $\rho_{S}$ is pure [28], such as the states in equations (7)-(10). They can be written as:

$$
\begin{aligned}
\rho_{S} & =|\psi\rangle\langle\psi|, \\
|\psi\rangle & =a|00\rangle+b|01\rangle+c|10\rangle+d|11\rangle,
\end{aligned}
$$

then equation (29) could be simplified to

$$
C(|\psi\rangle)=2|a d-b c|
$$

\section{RESULTS AND DISCUSSIONS}

In this section we give the calculated results of polarization correlations and concurrence with discussions. In all the calculations given here, the parameters $\lambda_{b b}=\lambda_{s s}=1.0$ unless otherwise specified. And the temperature is set as $T=0.1$ much higher than $T=0.02$ in

Ref. [1] in order to stress the generality of the algorithm and conclusion. The results for isolated systems $\lambda_{s b}=0.0$ are presented as a standard for comparison, in which there is no decoherence occurs. On the other hand, the case of $\lambda_{b b}=0.0$ will also be considered, in which there is a strong decoherence occurs and the subsystem initial state is beyond retrieval.

\section{A. Polarization correlation}

The polarization correlations of the isolated system are plotted in figures 1, 2] and 3, we see that the evolutions of the polarization correlations are periodic in time. Then we take into account the case that $\lambda_{b b}=0.0$, which means there is no coupling among bath spins, in order to highlight the effect of strong intra-bath coupling. In figure 4 , we show the pure 


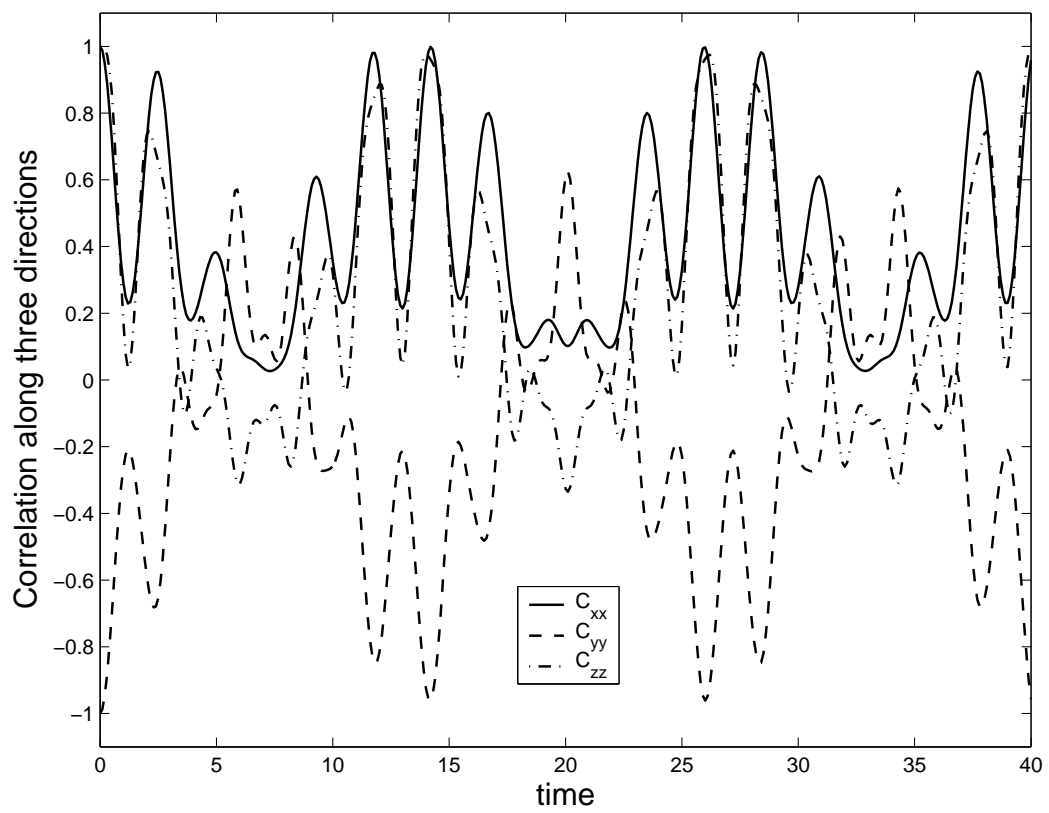

FIG. 1: Evolution for polarization correlation of the isolated subsystem, $C_{\alpha \alpha}=\left\langle\sigma_{\alpha}^{(1)} \sigma_{\alpha}^{(2)}\right\rangle-$ $\left\langle\sigma_{\alpha}^{(1)}\right\rangle\left\langle\sigma_{\alpha}^{(2)}\right\rangle, \alpha$ is $x, y$ or $z$, between the two spins in the subsystem. The initial state of the subsystem is $1 / \sqrt{2}(|00\rangle+|11\rangle)$.

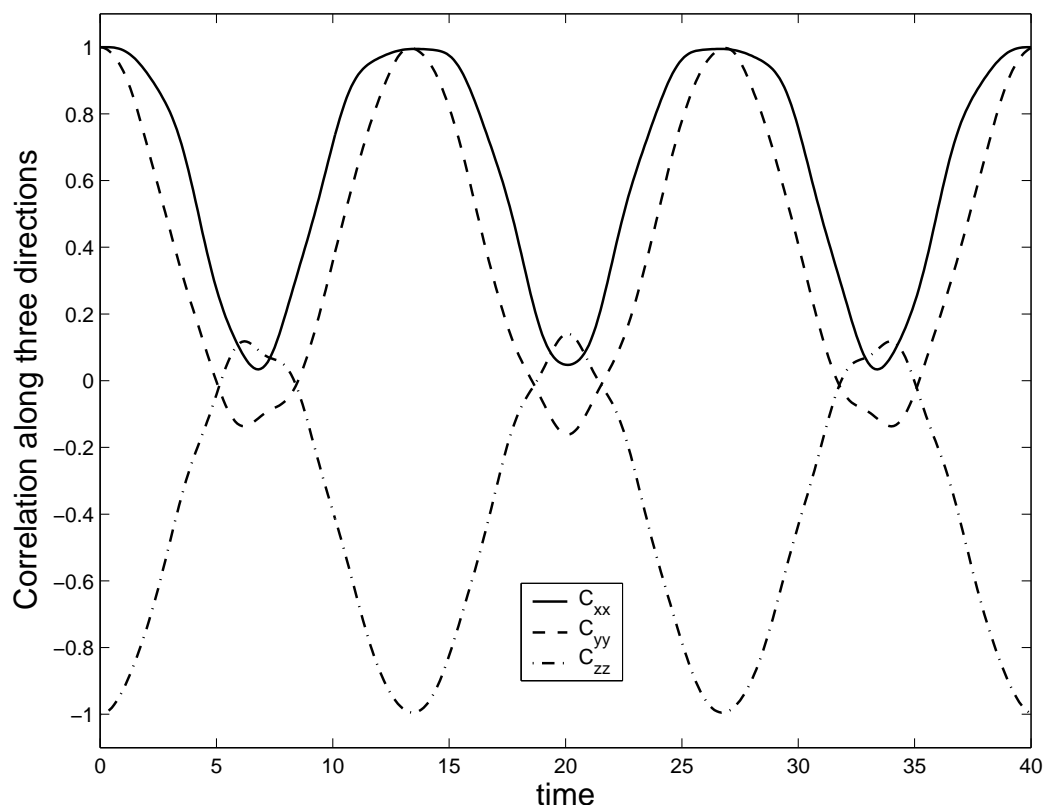

FIG. 2: Evolution for polarization correlation of the isolated subsystem, $C_{\alpha \alpha}=\left\langle\sigma_{\alpha}^{(1)} \sigma_{\alpha}^{(2)}\right\rangle-$ $\left\langle\sigma_{\alpha}^{(1)}\right\rangle\left\langle\sigma_{\alpha}^{(2)}\right\rangle, \alpha$ is $x, y$ or $z$, between the two spins in the subsystem. The initial state of the subsystem is $1 / \sqrt{2}(|01\rangle+|10\rangle)$. 


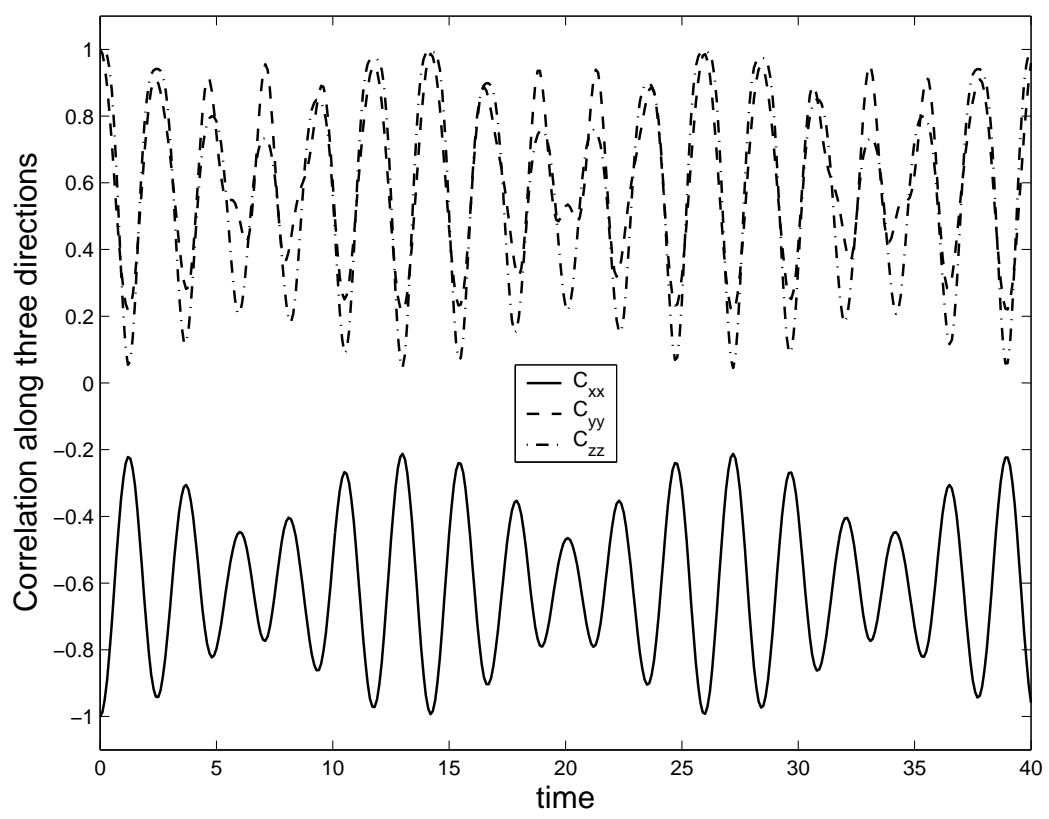

FIG. 3: Evolution for polarization correlation of the isolated subsystem, $C_{\alpha \alpha}=\left\langle\sigma_{\alpha}^{(1)} \sigma_{\alpha}^{(2)}\right\rangle-$ $\left\langle\sigma_{\alpha}^{(1)}\right\rangle\left\langle\sigma_{\alpha}^{(2)}\right\rangle, \alpha$ is $x, y$ or $z$, between the two spins in the subsystem. The initial state of the subsystem is $1 / \sqrt{2}(|00\rangle-|11\rangle)$.

destruction effect on polarization correlation of subsystem spins imposed by the bath spins $(m=6)$ to the open subsystem, where we neglect the intra-bath coupling strength. The initial state of the subsystem is $\left|\psi_{S}(0)\right\rangle^{2}=1 / \sqrt{2}(|01\rangle+|10\rangle)$.

Figure 5 through figure 13 are the polarization correlations when the subsystem coupled to the bath with different $\lambda_{b b}$. The corresponding results for isolated systems are also drawn on each figure as solid lines. In these figures, we have $m=4$ spins in the bath, though the number of bath spins is small, it still can give some information on the influence of bath coupling to the system. We also did calculations with more bath spins, $m=6$, show in figure 14,

It is clear from the figures that when the subsystem coupled to the bath, all the polarization correlations are different from the isolated systems. The difference reflects the influences of the bath and decoherence of the subsystem. However, as pointed out in reference [1] and 14] for the case of a single spin system, the coupling strength among environment spins can suppress the decoherence and partially restore the subsystem coherence. Figure 5 through figure 13 are plots of the polarization correlations as function of the time with different 


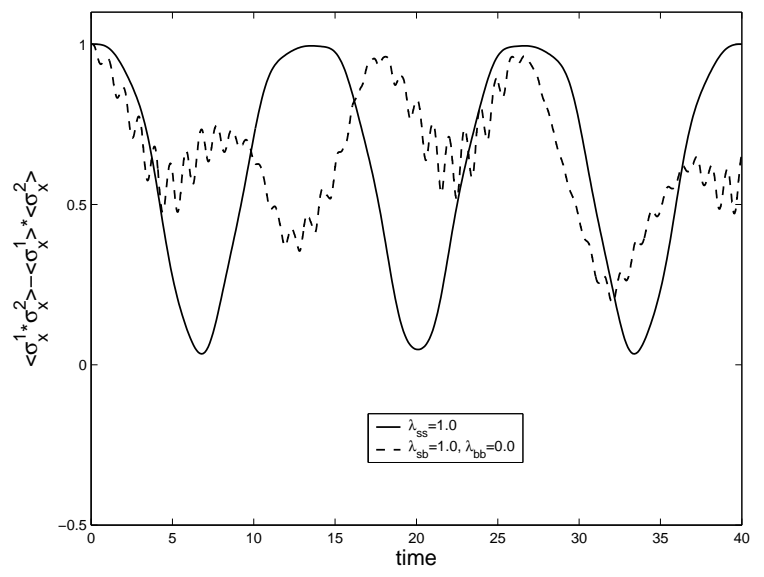

(a) $\left\langle\sigma_{x}^{1} \sigma_{x}^{2}\right\rangle-\left\langle\sigma_{x}^{1}\right\rangle\left\langle\sigma_{x}^{2}\right\rangle, \lambda_{b b}=0.0$

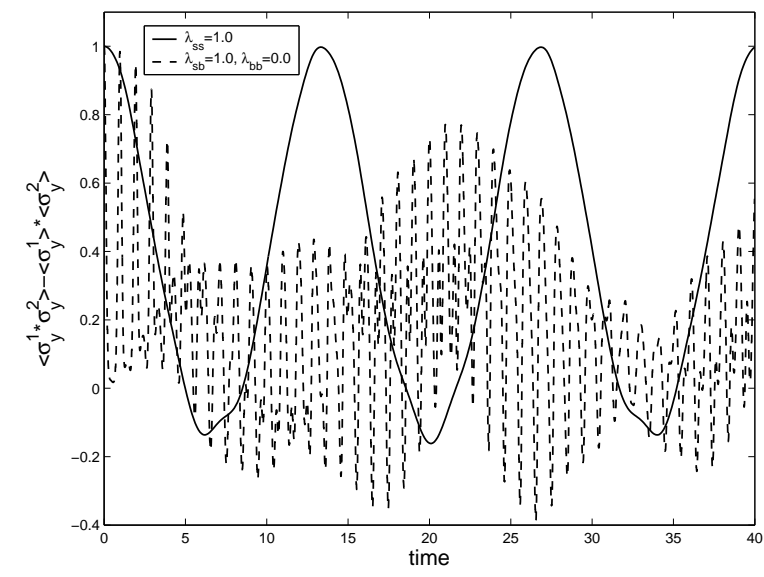

(b) $\left\langle\sigma_{y}^{1} \sigma_{y}^{2}\right\rangle-\left\langle\sigma_{y}^{1}\right\rangle\left\langle\sigma_{y}^{2}\right\rangle, \lambda_{b b}=0.0$

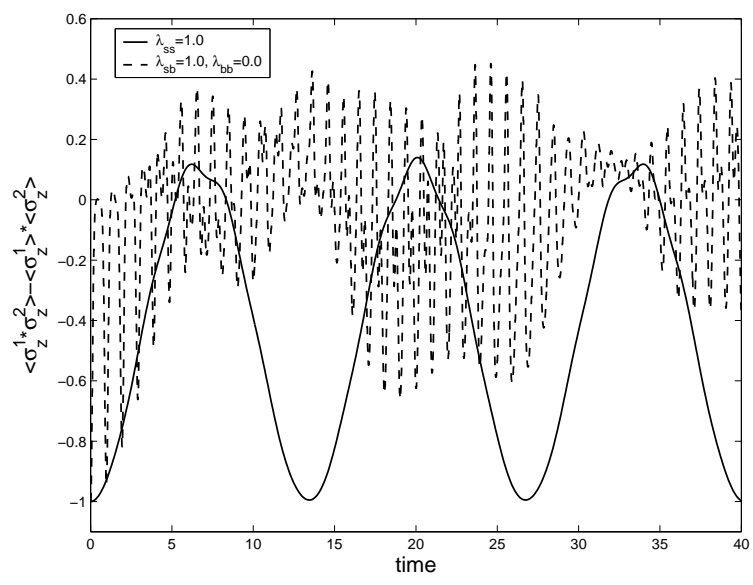

(c) $\left\langle\sigma_{z}^{1} \sigma_{z}^{2}\right\rangle-\left\langle\sigma_{z}^{1}\right\rangle\left\langle\sigma_{z}^{2}\right\rangle, \lambda_{b b}=0.0$

FIG. 4: The evolution of polarization correlation along three directions of the open subsystem. Where there are 6 spins in the bath and the initial state of the subsystem is $1 / \sqrt{2}(|10\rangle+|01\rangle)$ and $\lambda_{s s}=\lambda_{s b}=1.0, \lambda_{b b}=0.0$.

initial states and increasing intra-bath coupling strength. At weak intra-bath coupling, the results are drastically different from the isolated systems and similar with figure 4; as the intra-bath coupling is increased, the difference between the open subsystem and the isolated system becomes smaller; and at very strong strengths, the difference becomes very small especially in the case of $\left|\psi_{S}(0)\right\rangle^{2}$. The other two initial Bell states will be dissipated when time is elongated.

These results suggest that by changing the bath coupling strength, one can effectively control the subsystem coherence properties. The suppression of decoherence effect is also depends on the number of bath spins, it is found that the more bath spins, the less effective 


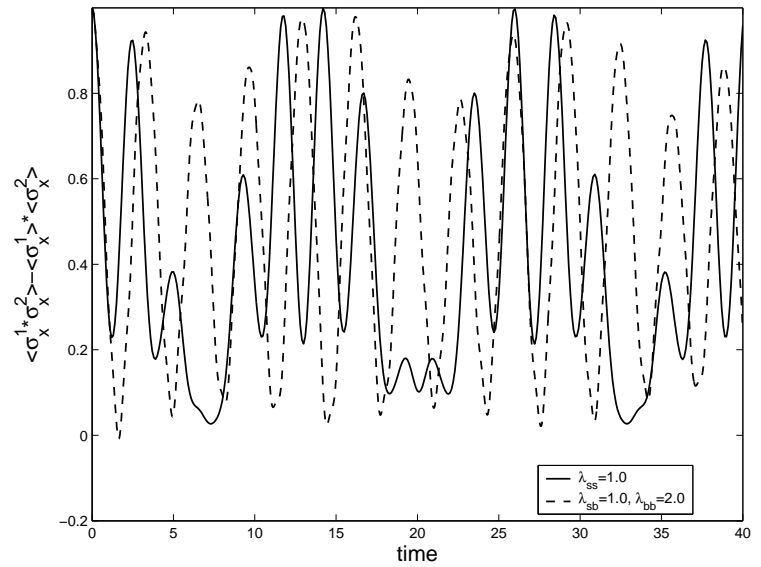

(a) $\lambda_{b b}=2.0$

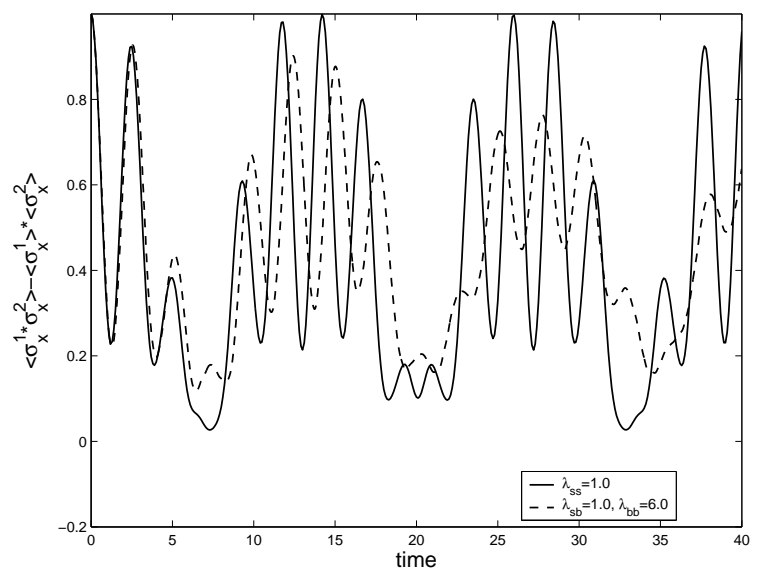

(c) $\lambda_{b b}=6.0$

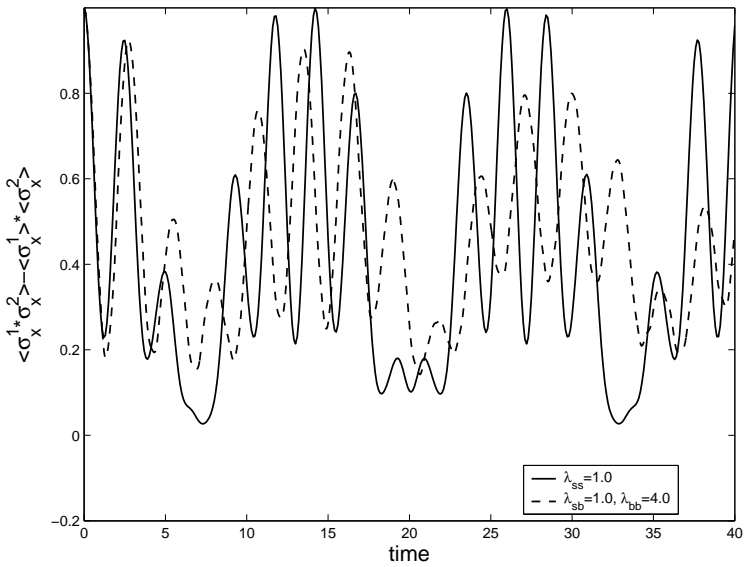

(b) $\lambda_{b b}=4.0$

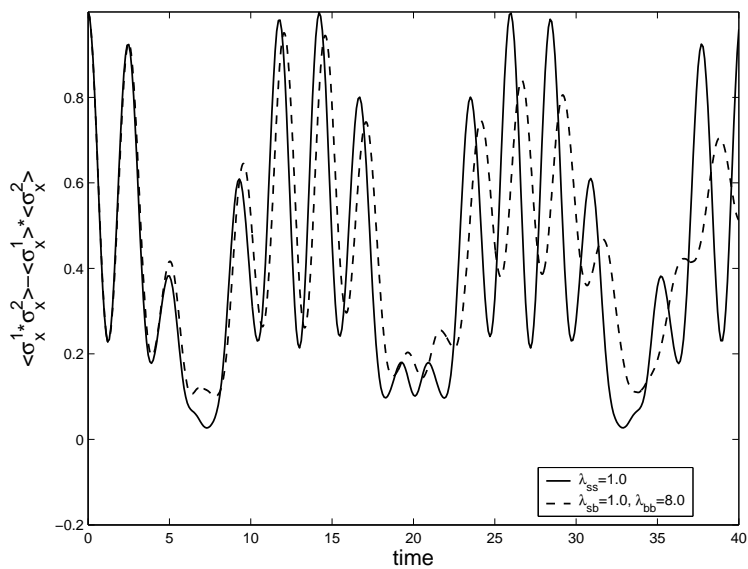

(d) $\lambda_{b b}=8.0$

FIG. 5: Evolution for polarization correlation along $\vec{x}$ direction of the open subsystem, $\left\langle\sigma_{x}^{1} \sigma_{x}^{2}\right\rangle-$ $\left\langle\sigma_{x}^{1}\right\rangle\left\langle\sigma_{x}^{2}\right\rangle$. The initial state of the subsystem is $1 / \sqrt{2}(|00\rangle+|11\rangle)$.

of suppression of the decoherence with the same bath coupling strength. To show this, we provide the results of $\lambda_{b b}=6.0$ and $\lambda_{b b}=10.0$ on the Bell state of $\left|\psi_{S}(0)\right\rangle^{2}=1 / \sqrt{2}(|01\rangle+$ $|10\rangle)$ in figure 14, where there are $m=6$ bath spins. The values of other parameters are the same as before. Through the comparison of figure 14(a) with figure 8(c), we see that when $m=4, \lambda_{b b}=6.0$ is almost sufficient to recover the isolated case except a slightly elongated period (see figure 8(c) , but when $m$ is increased to 6 , the coupling strength need to be increased at least to $\lambda_{b b}=10.0$ to get the same effect (see figure 14(b)). The same feature also happens to the evolution of $C_{y y}$ and $C_{z z}$ which can be seen by comparing figure 14(c) with figure 9(c) and figure 14(e) with figure 10(c). If we go on to increase the coupling strength $\lambda_{b b}$, we can suppress the decoherence and disentanglement effect due to 


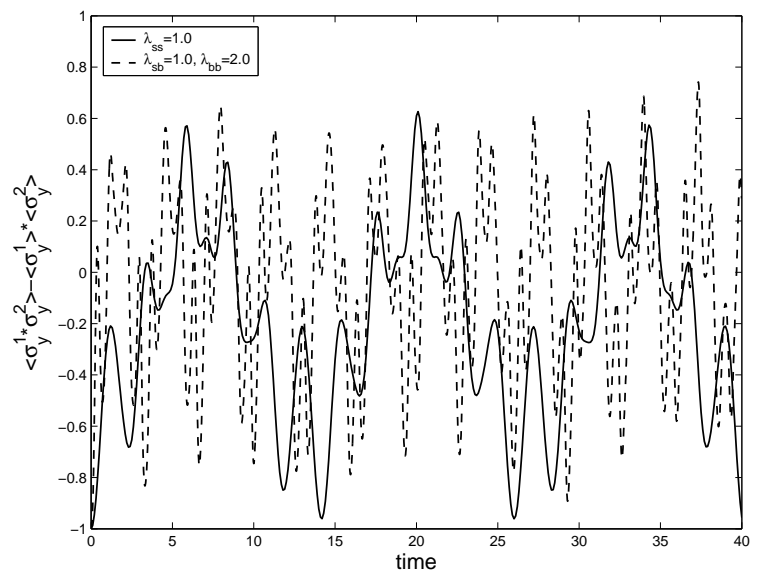

(a) $\lambda_{b b}=2.0$

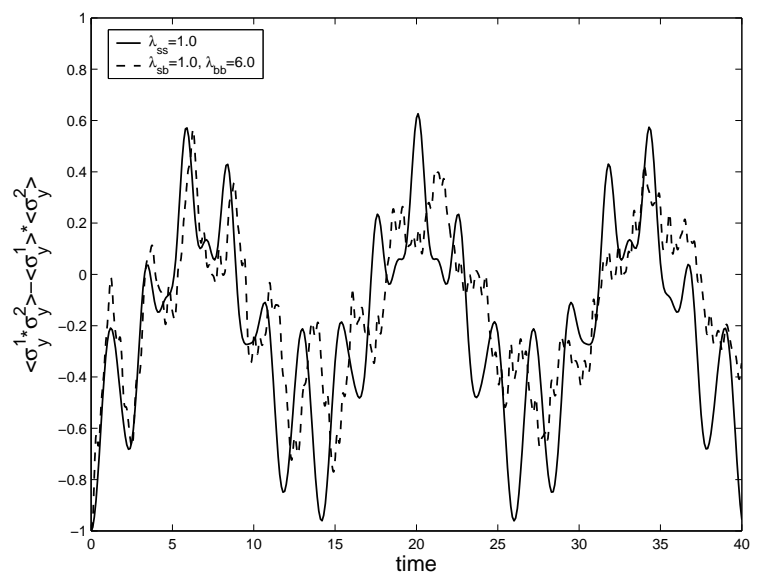

(c) $\lambda_{b b}=6.0$

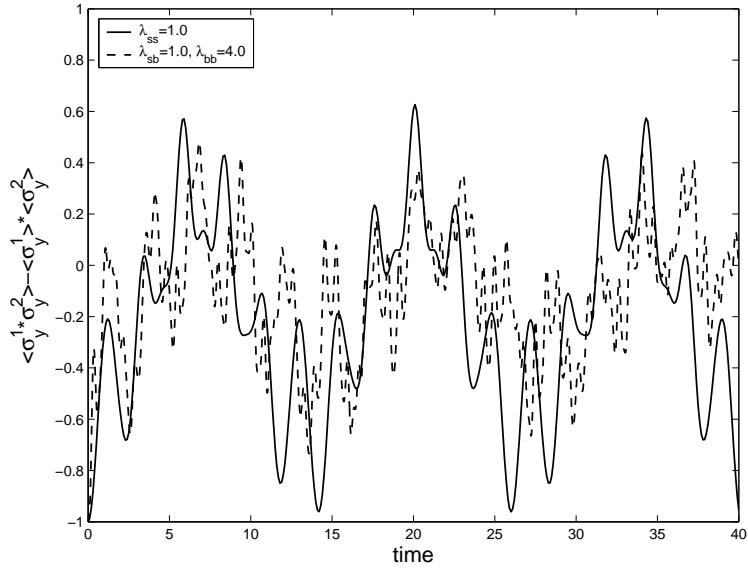

(b) $\lambda_{b b}=4.0$

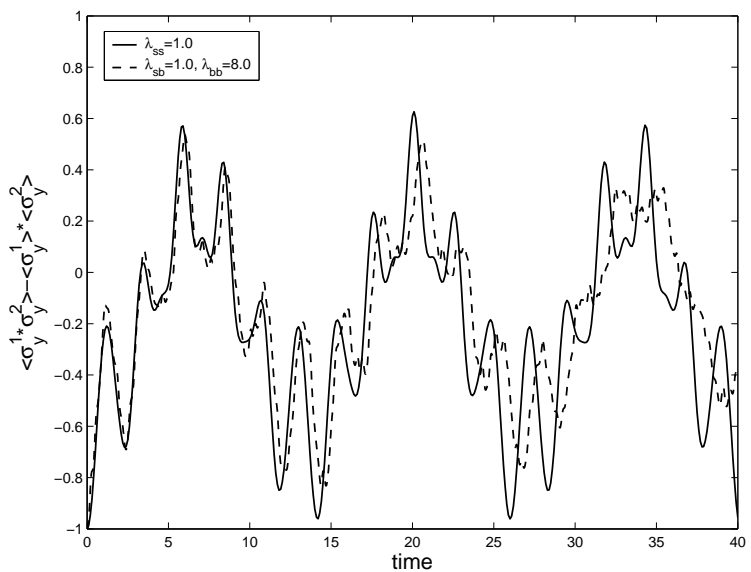

(d) $\lambda_{b b}=8.0$

FIG. 6: Evolution for polarization correlation along $\vec{y}$ direction of the open subsystem, $\left\langle\sigma_{y}^{1} \sigma_{y}^{2}\right\rangle-$ $\left\langle\sigma_{y}^{1}\right\rangle\left\langle\sigma_{y}^{2}\right\rangle$. The initial state of the subsystem is $1 / \sqrt{2}(|00\rangle+|11\rangle)$.

the bath with more spins. In figure 15, we compare the results of the cases of $\lambda_{b b}=4.0$ and $\lambda_{b b}=24.0$, in which the subsystem seems to be decoupled from the bath; the initial state is also $1 / \sqrt{2}(|01\rangle+|10\rangle)$. 


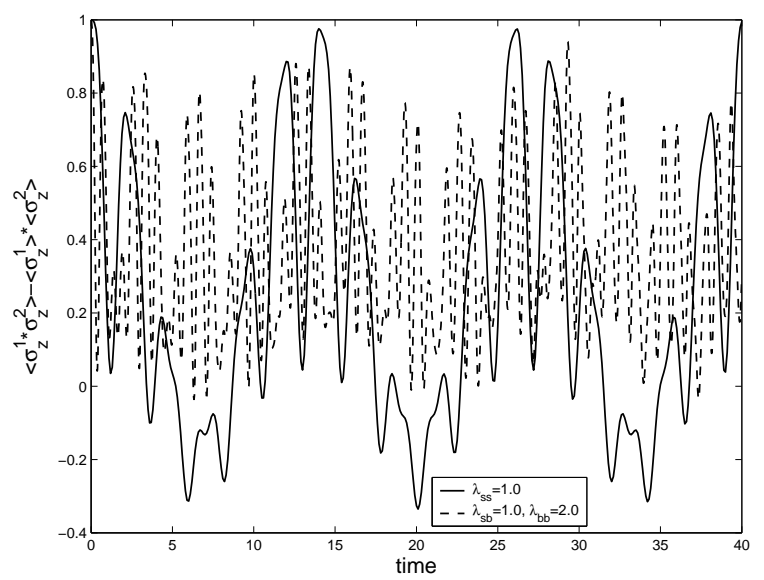

(a) $\lambda_{b b}=2.0$

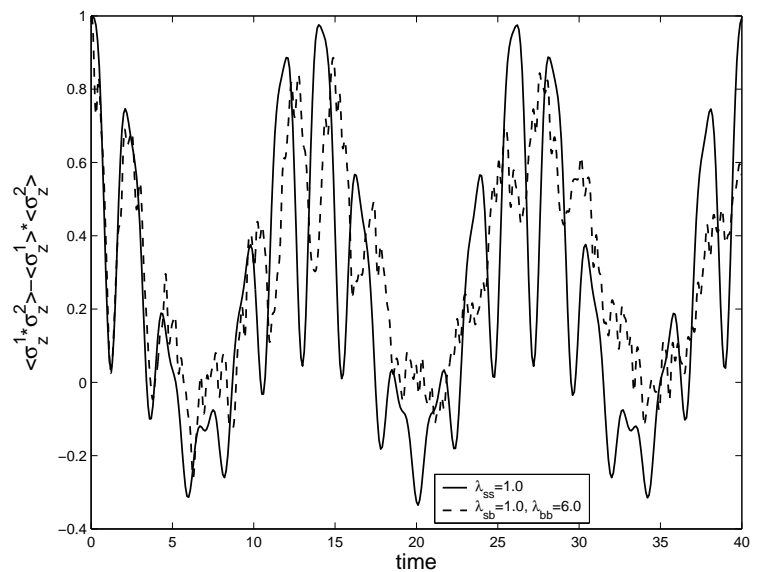

(c) $\lambda_{b b}=6.0$

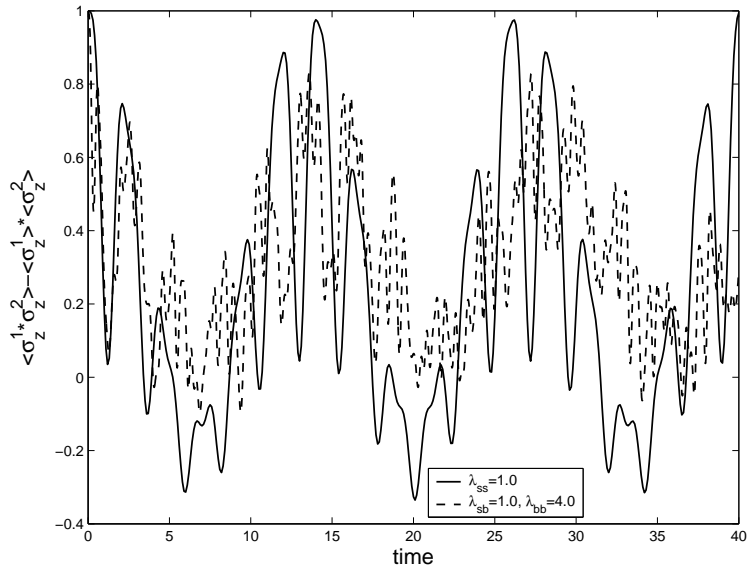

(b) $\lambda_{b b}=4.0$

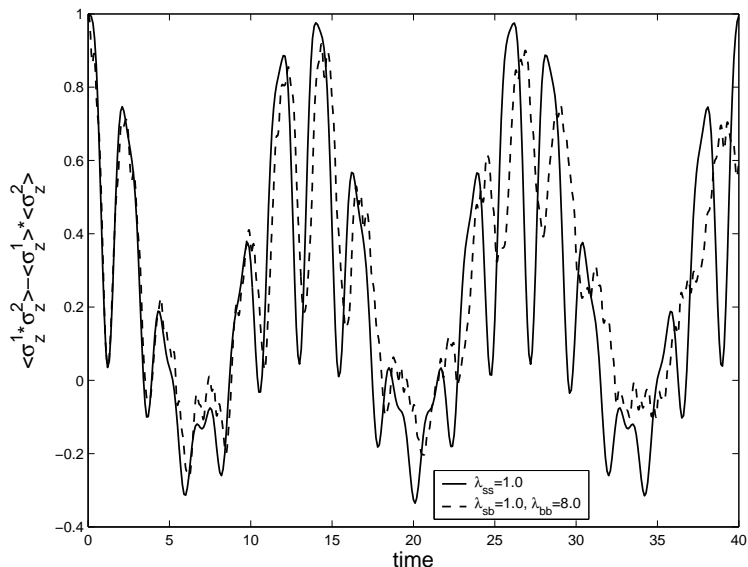

(d) $\lambda_{b b}=8.0$

FIG. 7: Evolution for polarization correlation along $\vec{z}$ direction of the open subsystem, $\left\langle\sigma_{z}^{1} \sigma_{z}^{2}\right\rangle-$ $\left\langle\sigma_{z}^{1}\right\rangle\left\langle\sigma_{z}^{2}\right\rangle$. The initial state of the subsystem is $1 / \sqrt{2}(|00\rangle+|11\rangle)$. 


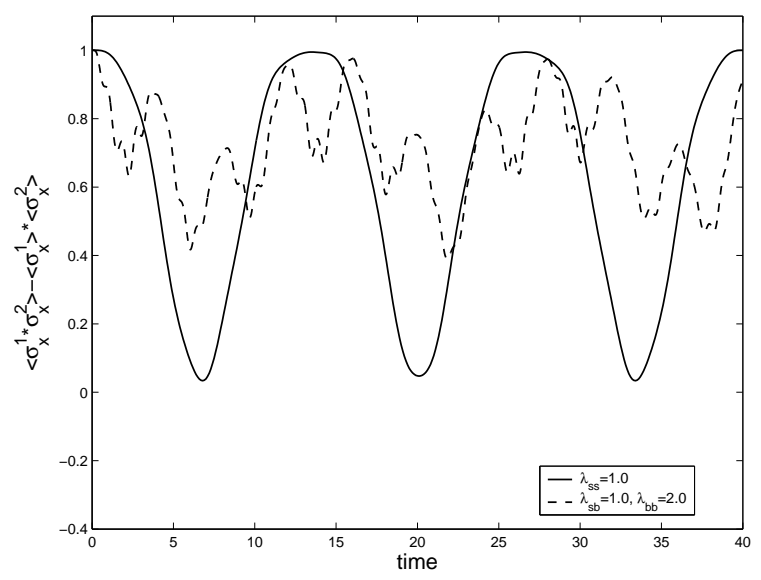

(a) $\lambda_{b b}=2.0$

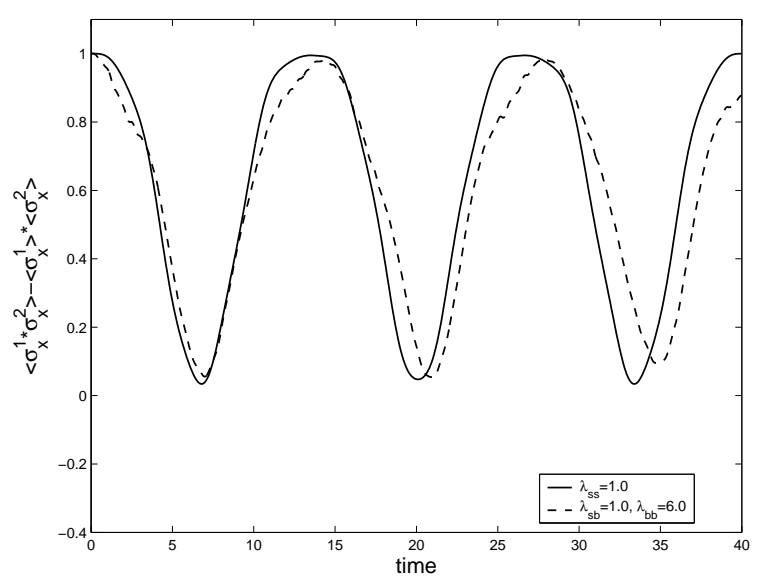

(c) $\lambda_{b b}=6.0$

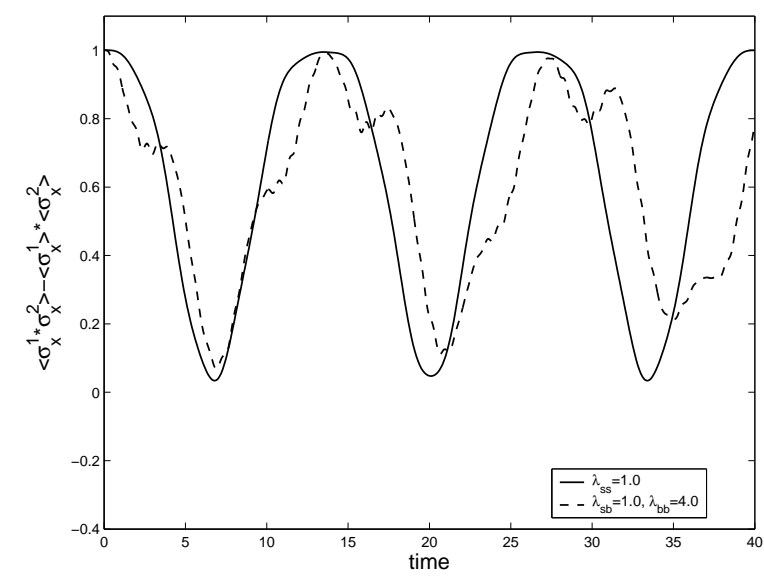

(b) $\lambda_{b b}=4.0$

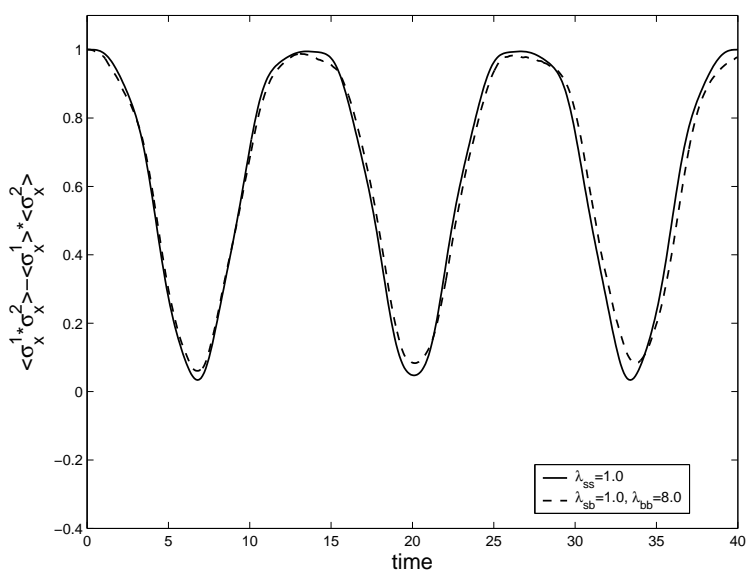

(d) $\lambda_{b b}=8.0$

FIG. 8: Evolution for polarization correlation along $\vec{x}$ direction of the open subsystem, $\left\langle\sigma_{x}^{1} \sigma_{x}^{2}\right\rangle-$ $\left\langle\sigma_{x}^{1}\right\rangle\left\langle\sigma_{x}^{2}\right\rangle$. The initial state of the subsystem is $1 / \sqrt{2}(|01\rangle+|10\rangle)$. 


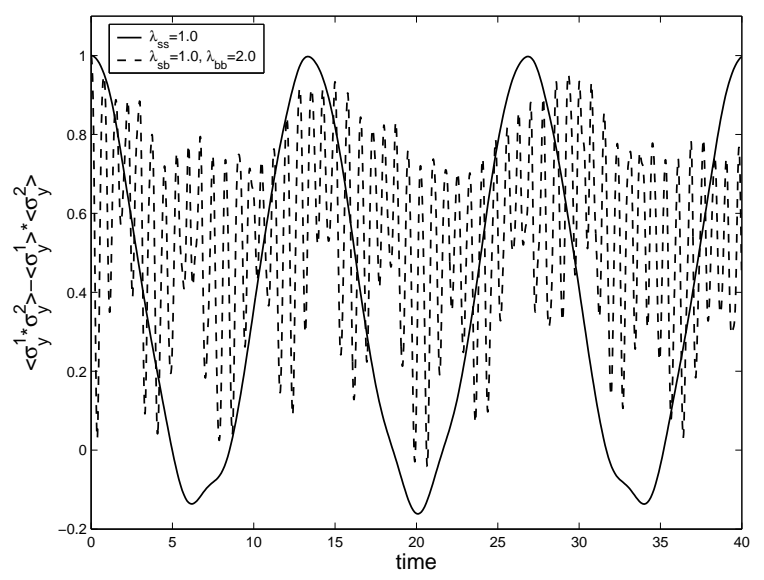

(a) $\lambda_{b b}=2.0$

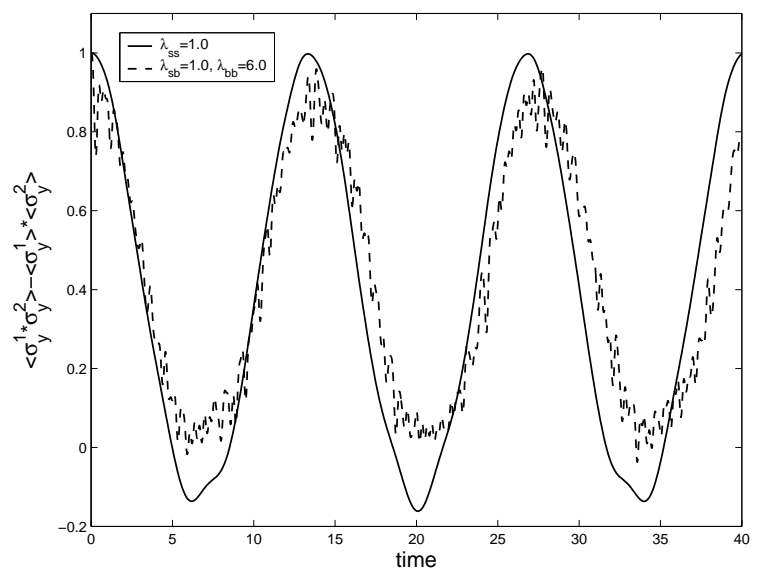

(c) $\lambda_{b b}=6.0$

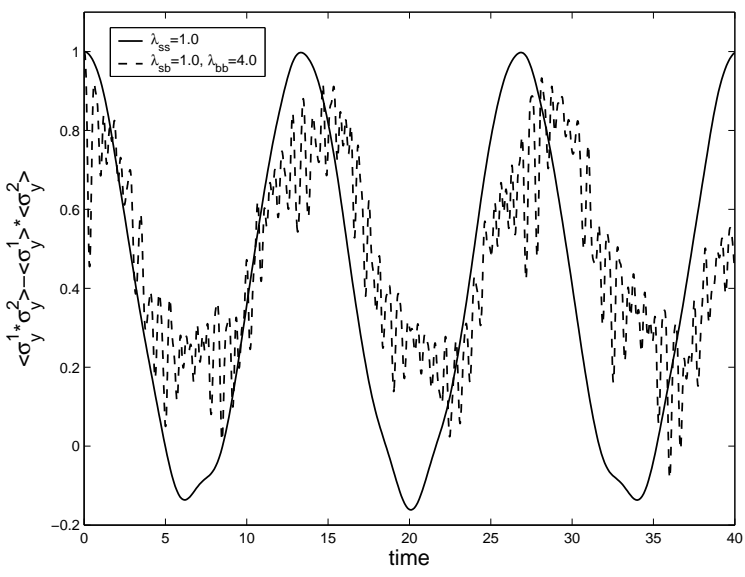

(b) $\lambda_{b b}=4.0$

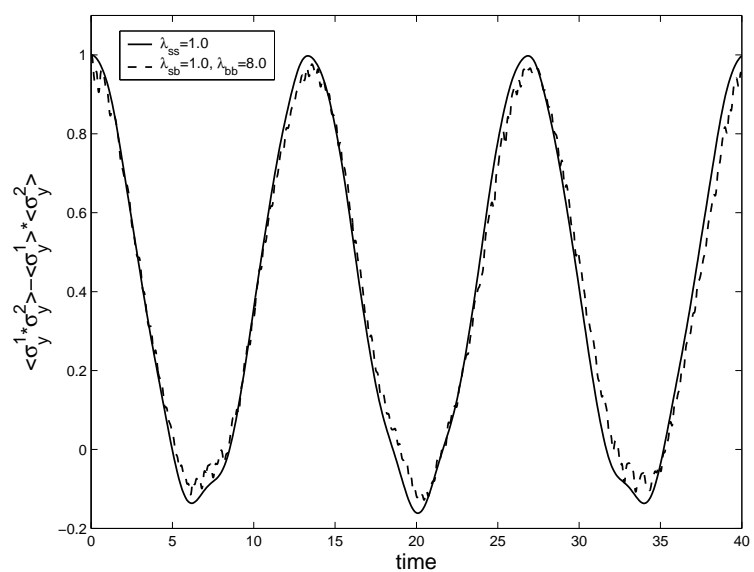

(d) $\lambda_{b b}=8.0$

FIG. 9: Evolution for polarization correlation along $\vec{y}$ direction of the open subsystem, $\left\langle\sigma_{y}^{1} \sigma_{y}^{2}\right\rangle-$ $\left\langle\sigma_{y}^{1}\right\rangle\left\langle\sigma_{y}^{2}\right\rangle$. The initial state of the subsystem is $1 / \sqrt{2}(|01\rangle+|10\rangle)$. 


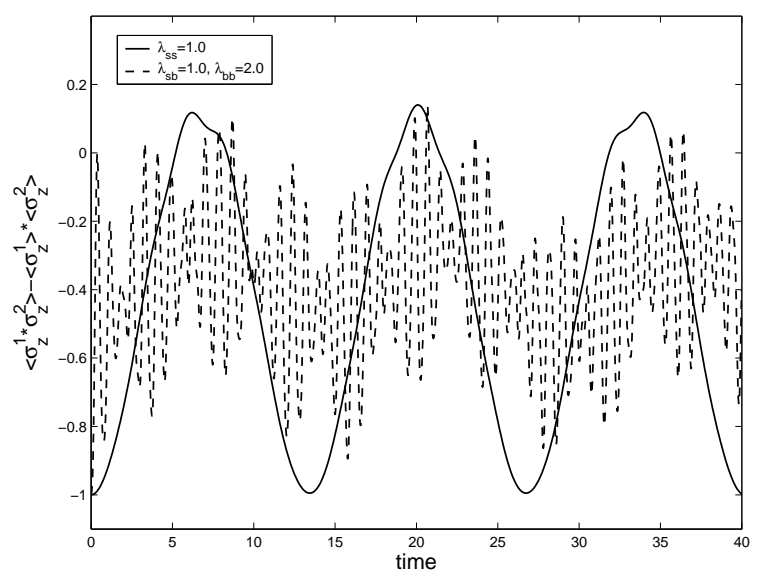

(a) $\lambda_{b b}=2.0$

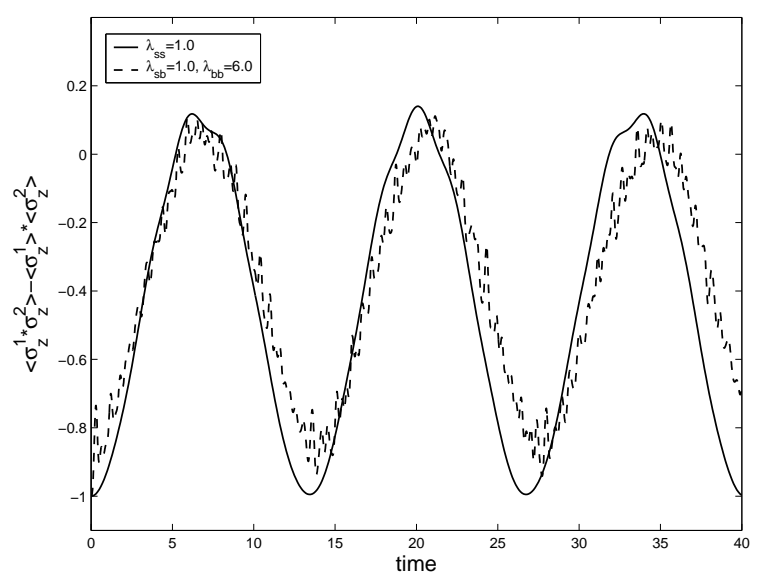

(c) $\lambda_{b b}=6.0$

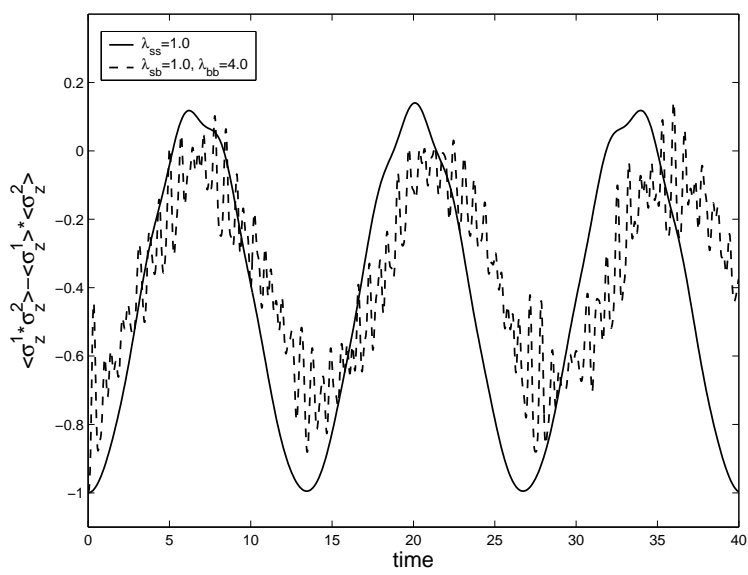

(b) $\lambda_{b b}=4.0$

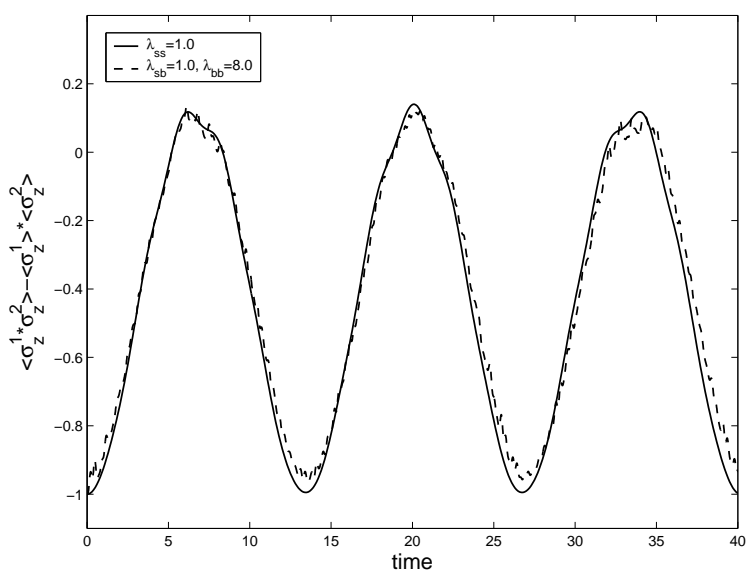

(d) $\lambda_{b b}=8.0$

FIG. 10: Evolution for polarization correlation along $\vec{z}$ direction of the open subsystem, $\left\langle\sigma_{z}^{1} \sigma_{z}^{2}\right\rangle-$ $\left\langle\sigma_{z}^{1}\right\rangle\left\langle\sigma_{z}^{2}\right\rangle$. The initial state of the subsystem is $1 / \sqrt{2}(|01\rangle+|10\rangle)$. 


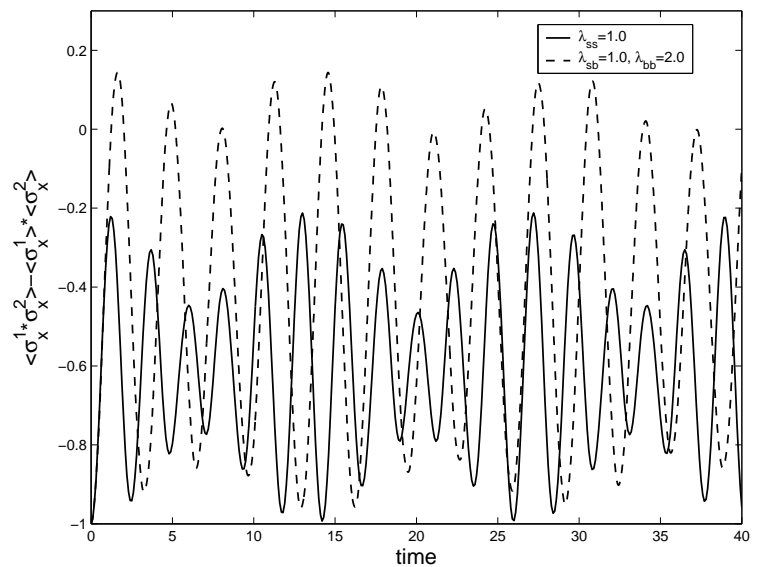

(a) $\lambda_{b b}=2.0$

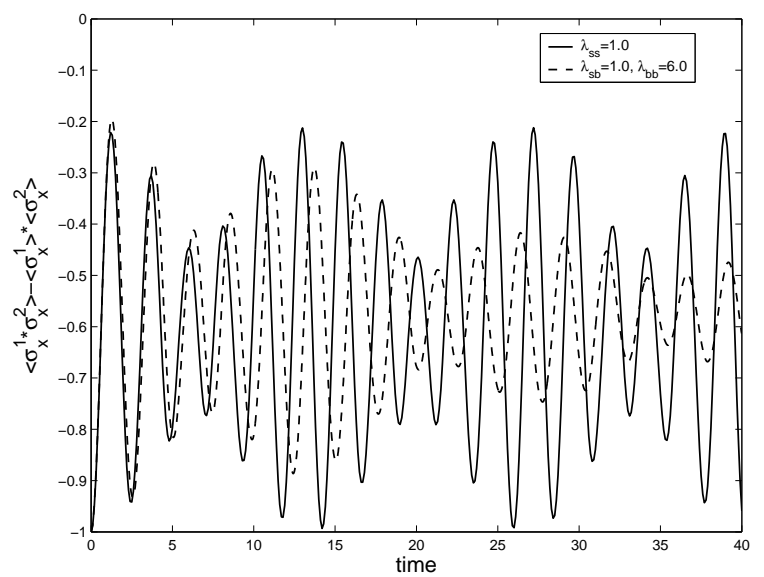

(c) $\lambda_{b b}=6.0$

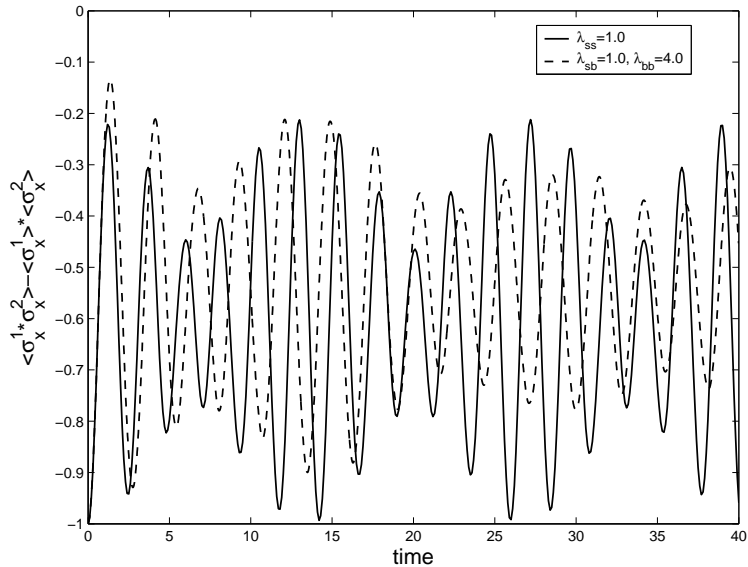

(b) $\lambda_{b b}=4.0$

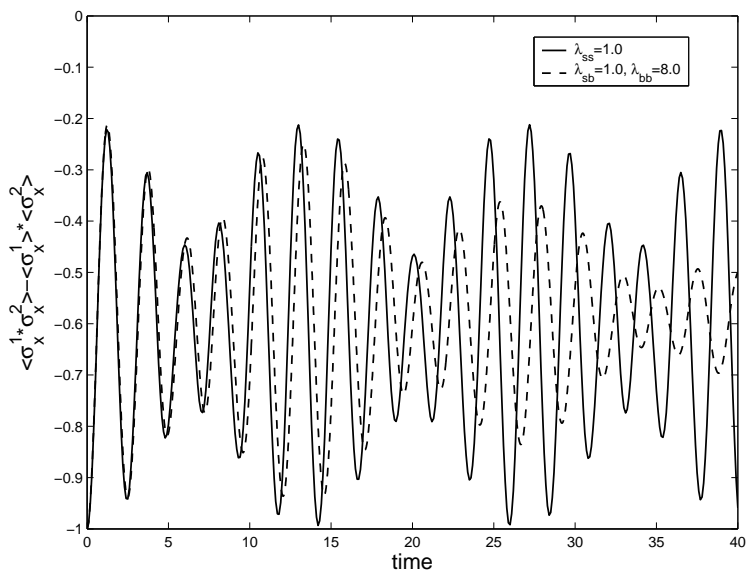

(d) $\lambda_{b b}=8.0$

FIG. 11: Evolution for polarization correlation along $\vec{x}$ direction of the open subsystem, $\left\langle\sigma_{x}^{1} \sigma_{x}^{2}\right\rangle-$ $\left\langle\sigma_{x}^{1}\right\rangle\left\langle\sigma_{x}^{2}\right\rangle$. The initial state of the subsystem is $1 / \sqrt{2}(|11\rangle-|00\rangle)$. 


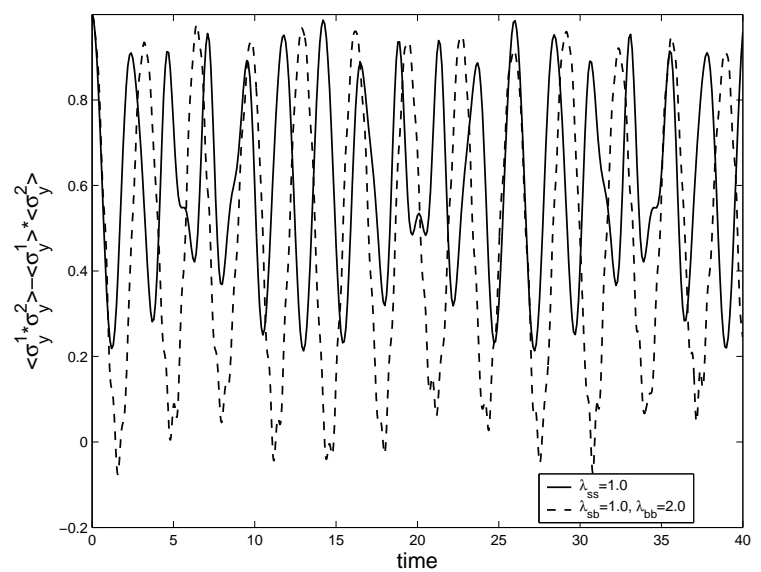

(a) $\lambda_{b b}=2.0$

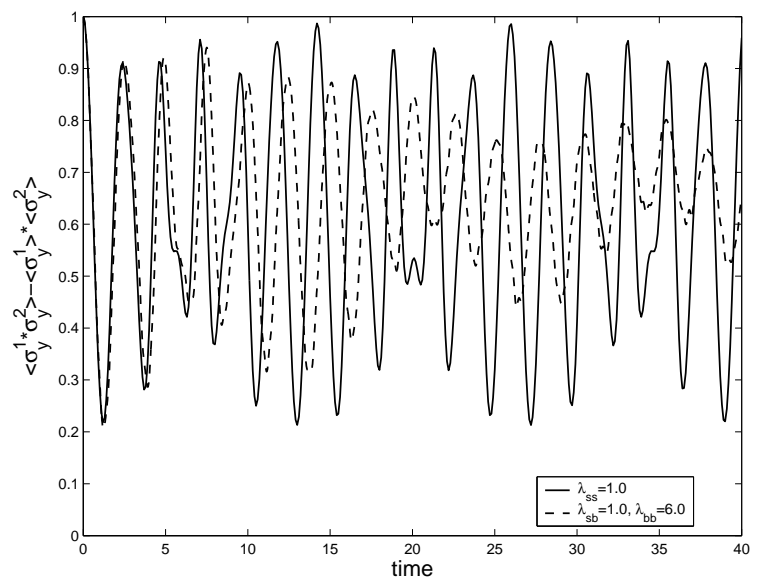

(c) $\lambda_{b b}=6.0$

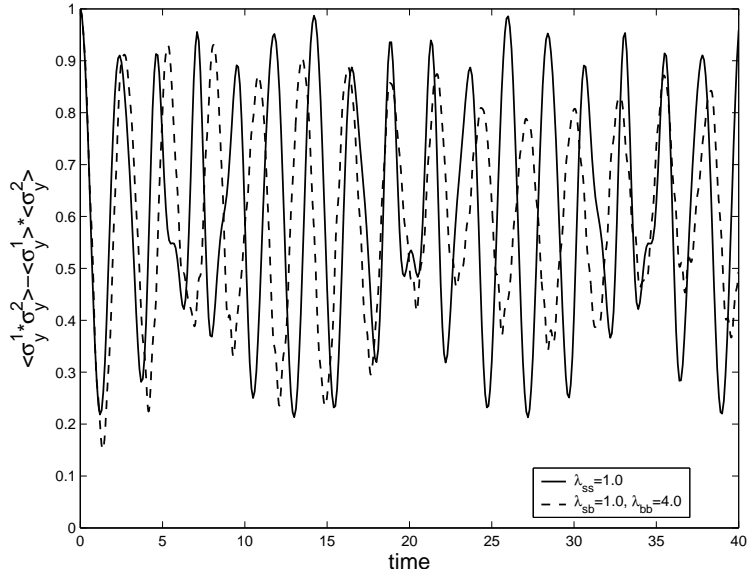

(b) $\lambda_{b b}=4.0$

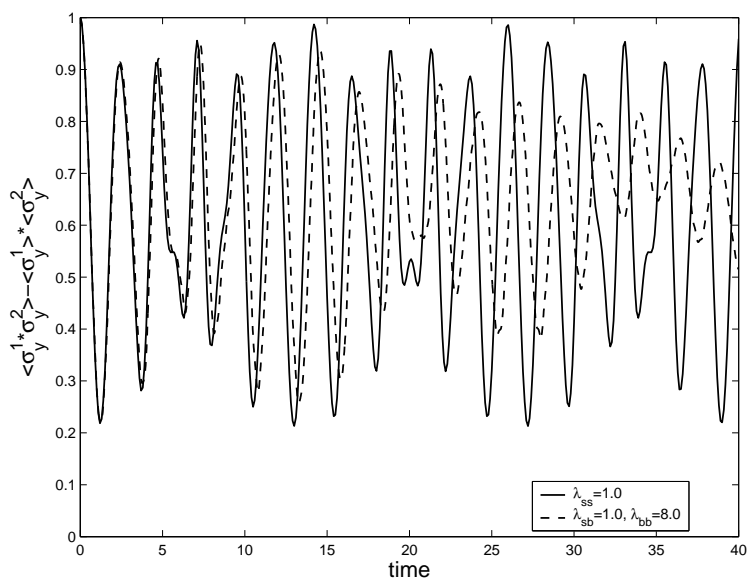

(d) $\lambda_{b b}=8.0$

FIG. 12: Evolution for polarization correlation along $\vec{y}$ direction of the open subsystem, $\left\langle\sigma_{y}^{1} \sigma_{y}^{2}\right\rangle-$ $\left\langle\sigma_{y}^{1}\right\rangle\left\langle\sigma_{y}^{2}\right\rangle$. The initial state of the subsystem is $1 / \sqrt{2}(|11\rangle-|00\rangle)$. 


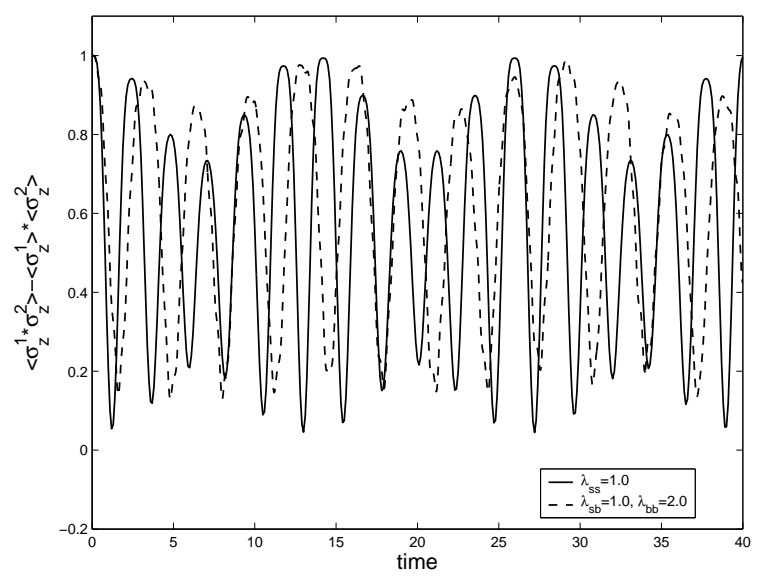

(a) $\lambda_{b b}=2.0$

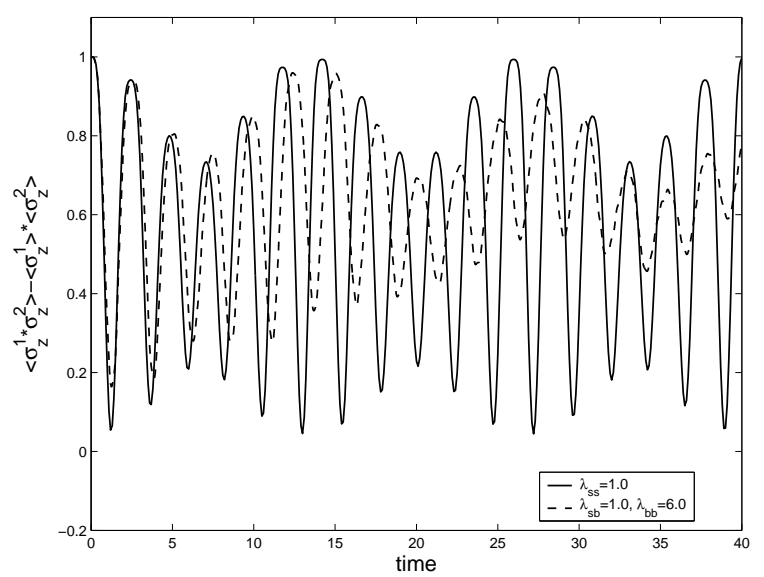

(c) $\lambda_{b b}=6.0$

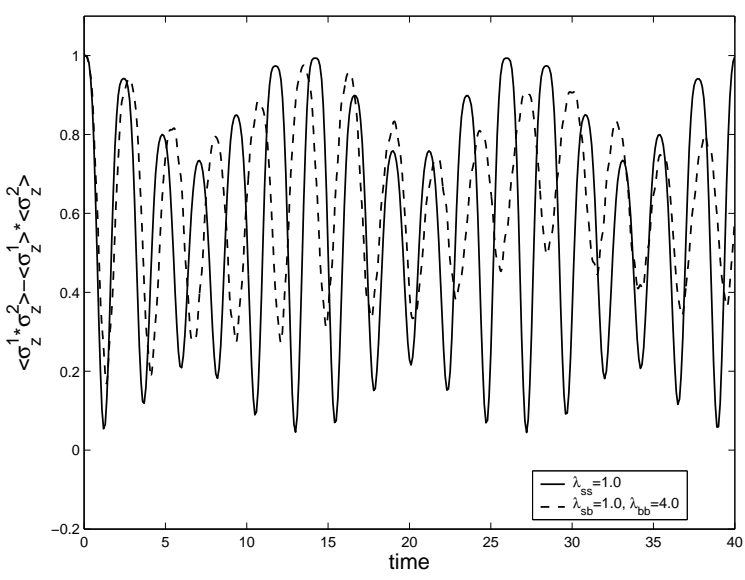

(b) $\lambda_{b b}=4.0$

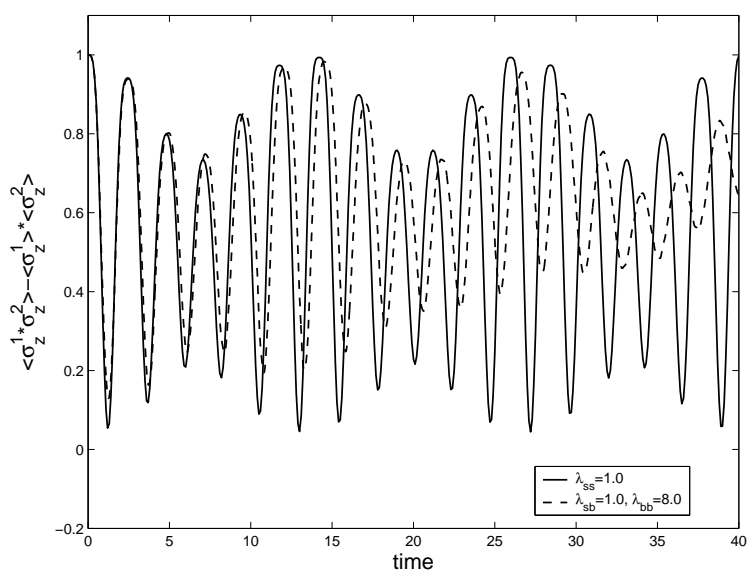

(d) $\lambda_{b b}=8.0$

FIG. 13: Evolution for polarization correlation along $\vec{z}$ direction of the open subsystem, $\left\langle\sigma_{z}^{1} \sigma_{z}^{2}\right\rangle-$ $\left\langle\sigma_{z}^{1}\right\rangle\left\langle\sigma_{z}^{2}\right\rangle$. The initial state of the subsystem is $1 / \sqrt{2}(|11\rangle-|00\rangle)$. 

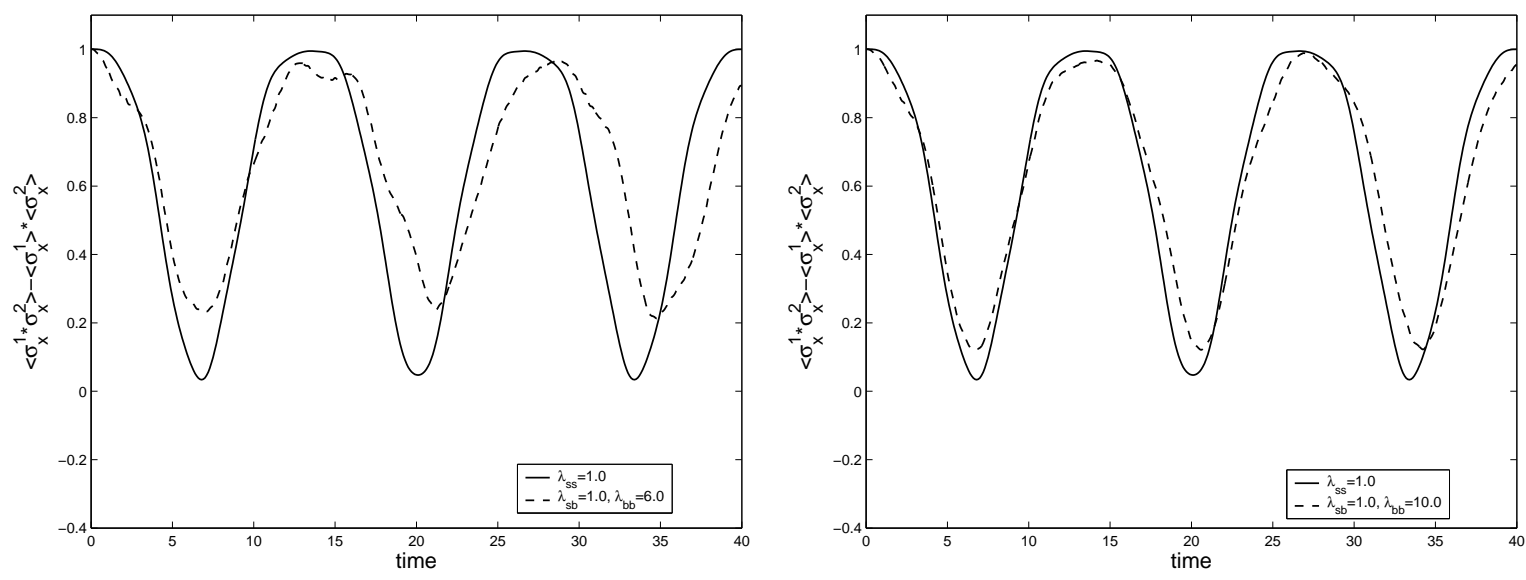

(a) $\left\langle\sigma_{x}^{1} \sigma_{x}^{2}\right\rangle-\left\langle\sigma_{x}^{1}\right\rangle\left\langle\sigma_{x}^{2}\right\rangle, \lambda_{b b}=6.0$

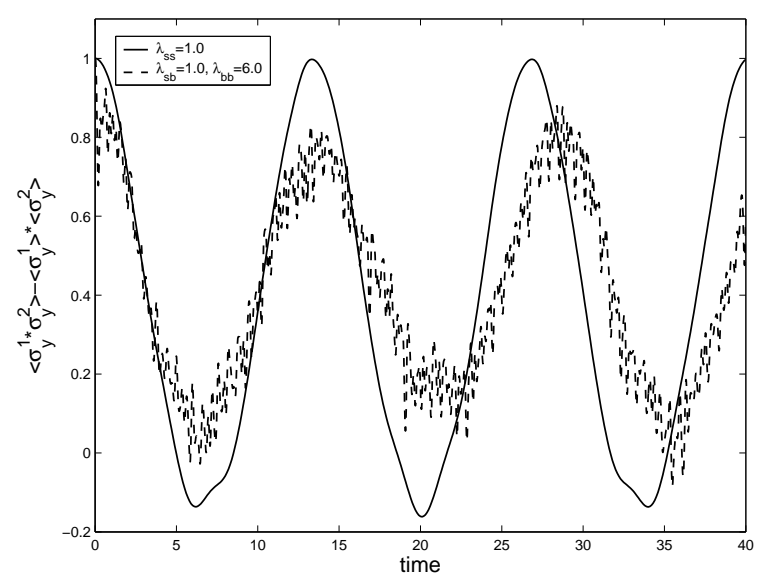

(b) $\left\langle\sigma_{x}^{1} \sigma_{x}^{2}\right\rangle-\left\langle\sigma_{x}^{1}\right\rangle\left\langle\sigma_{x}^{2}\right\rangle, \lambda_{b b}=10.0$

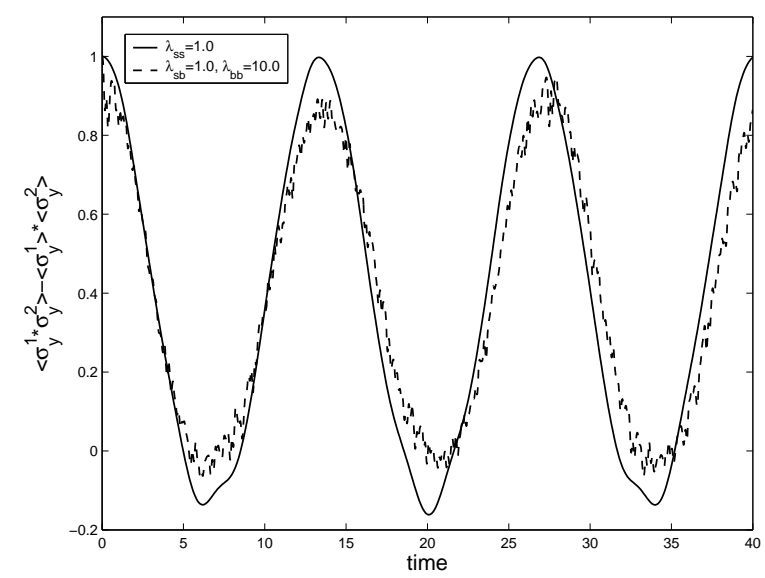

(c) $\left\langle\sigma_{y}^{1} \sigma_{y}^{2}\right\rangle-\left\langle\sigma_{y}^{1}\right\rangle\left\langle\sigma_{y}^{2}\right\rangle, \lambda_{b b}=6.0$

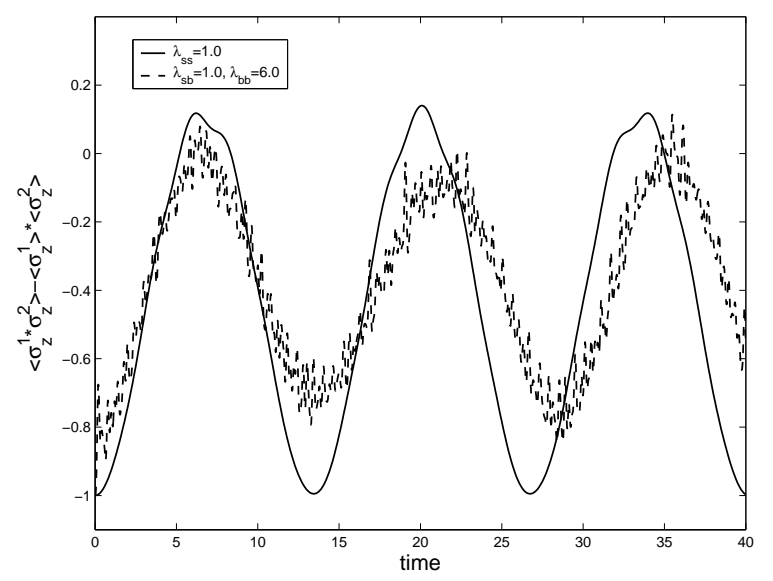

(d) $\left\langle\sigma_{y}^{1} \sigma_{y}^{2}\right\rangle-\left\langle\sigma_{y}^{1}\right\rangle\left\langle\sigma_{y}^{2}\right\rangle, \lambda_{b b}=10.0$

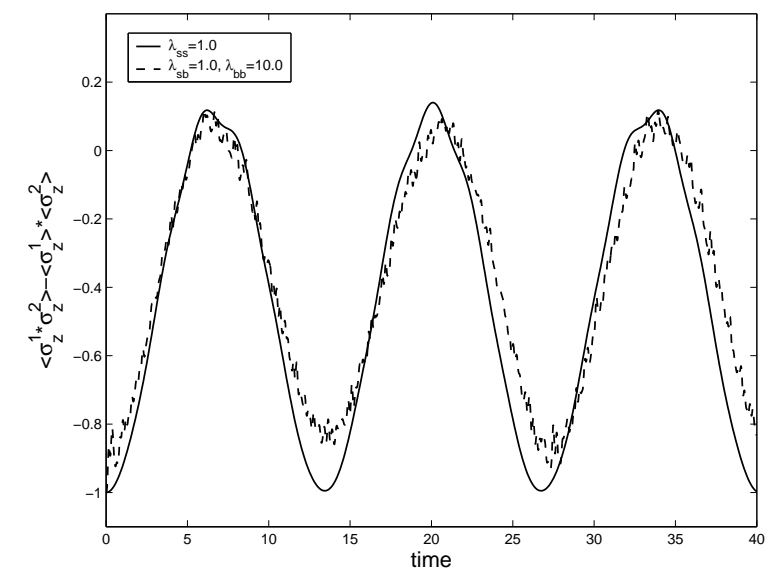

(e) $\left\langle\sigma_{z}^{1} \sigma_{z}^{2}\right\rangle-\left\langle\sigma_{z}^{1}\right\rangle\left\langle\sigma_{z}^{2}\right\rangle, \lambda_{b b}=6.0$

(f) $\left\langle\sigma_{z}^{1} \sigma_{z}^{2}\right\rangle-\left\langle\sigma_{z}^{1}\right\rangle\left\langle\sigma_{z}^{2}\right\rangle, \lambda_{b b}=10.0$

FIG. 14: Evolution for polarization correlations along three directions of the open subsystem.

Where there are 6 spins in the bath and the initial state of the subsystem is $1 / \sqrt{2}(|01\rangle+|10\rangle)$. 

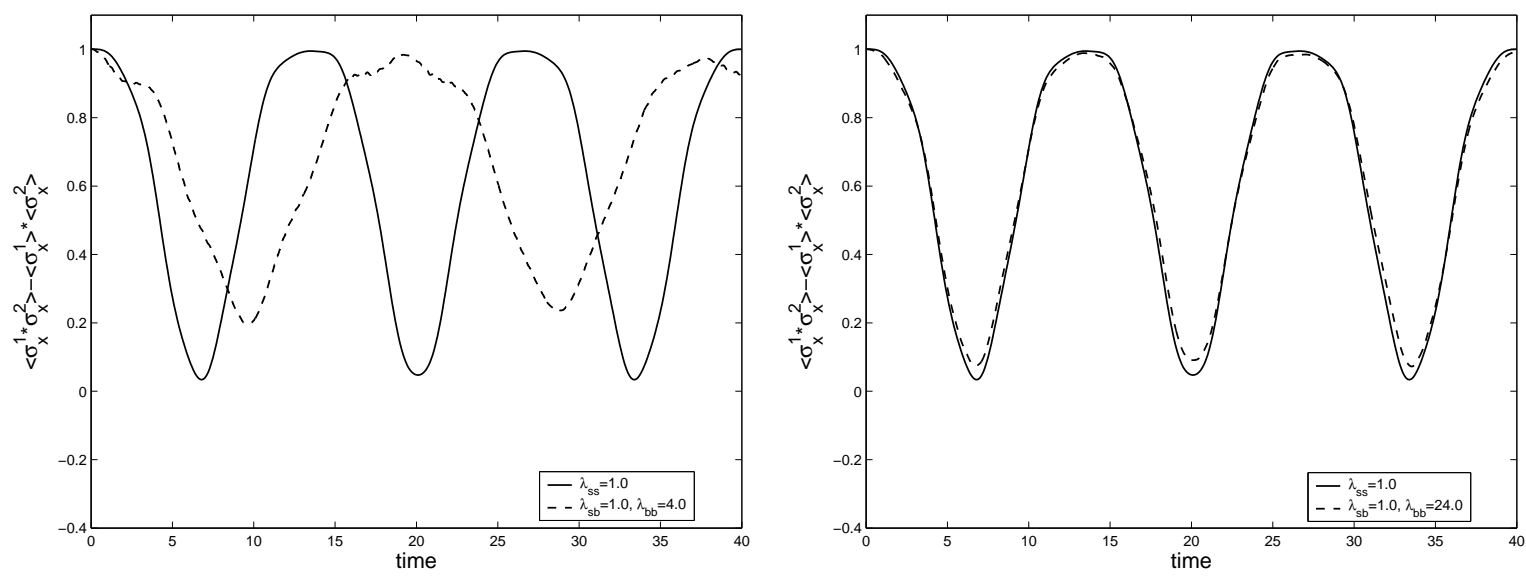

(a) $\left\langle\sigma_{x}^{1} \sigma_{x}^{2}\right\rangle-\left\langle\sigma_{x}^{1}\right\rangle\left\langle\sigma_{x}^{2}\right\rangle, \lambda_{b b}=4.0$

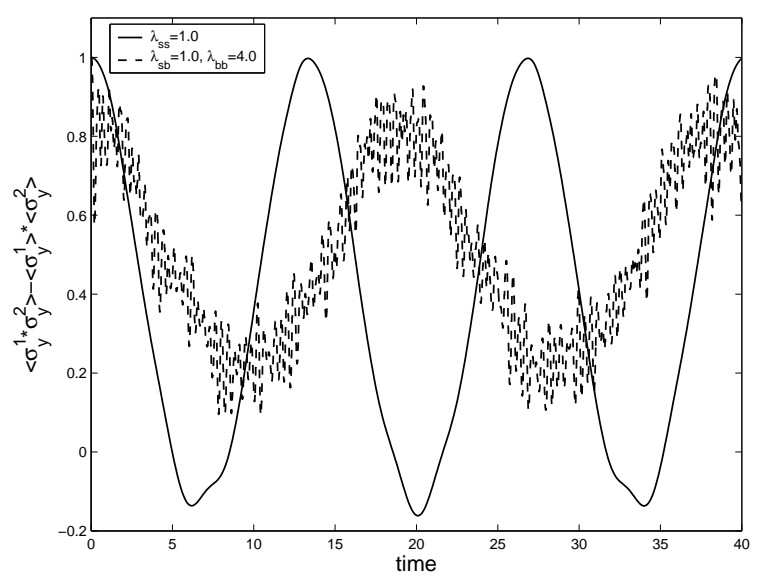

(b) $\left\langle\sigma_{x}^{1} \sigma_{x}^{2}\right\rangle-\left\langle\sigma_{x}^{1}\right\rangle\left\langle\sigma_{x}^{2}\right\rangle, \lambda_{b b}=24.0$

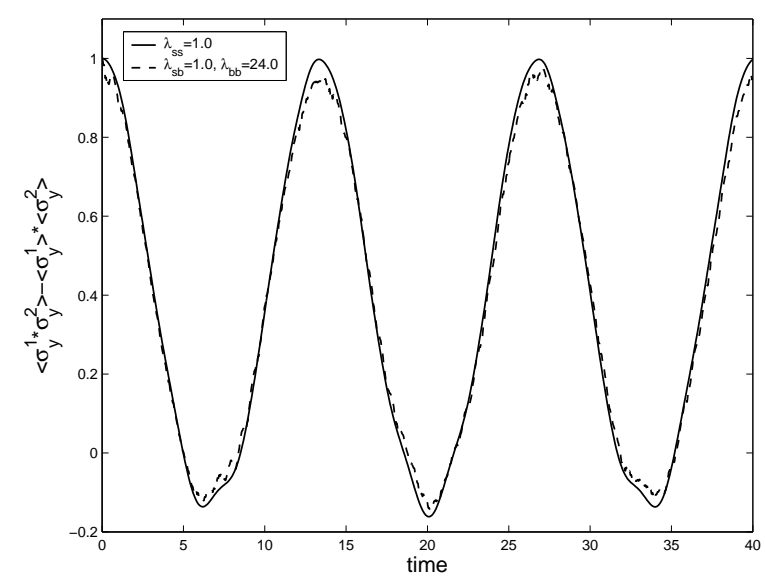

(c) $\left\langle\sigma_{y}^{1} \sigma_{y}^{2}\right\rangle-\left\langle\sigma_{y}^{1}\right\rangle\left\langle\sigma_{y}^{2}\right\rangle, \lambda_{b b}=4.0$

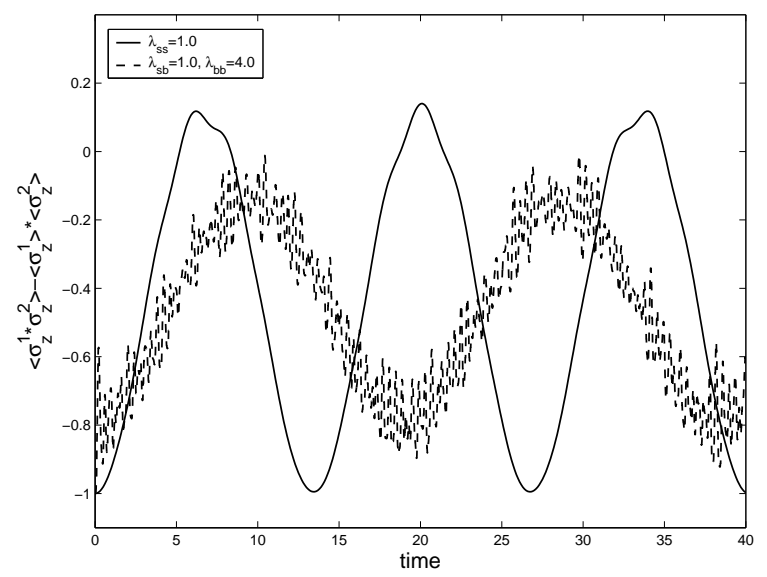

(d) $\left\langle\sigma_{y}^{1} \sigma_{y}^{2}\right\rangle-\left\langle\sigma_{y}^{1}\right\rangle\left\langle\sigma_{y}^{2}\right\rangle, \lambda_{b b}=24.0$

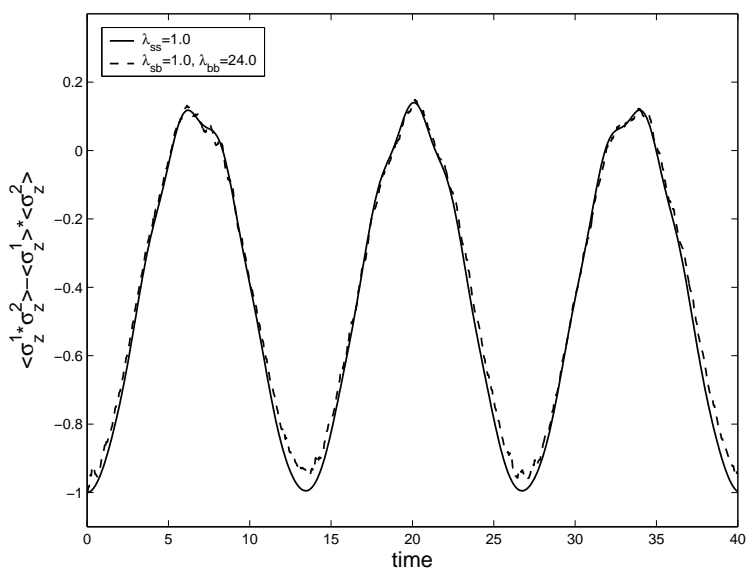

(e) $\left\langle\sigma_{z}^{1} \sigma_{z}^{2}\right\rangle-\left\langle\sigma_{z}^{1}\right\rangle\left\langle\sigma_{z}^{2}\right\rangle, \lambda_{b b}=4.0$

(f) $\left\langle\sigma_{z}^{1} \sigma_{z}^{2}\right\rangle-\left\langle\sigma_{z}^{1}\right\rangle\left\langle\sigma_{z}^{2}\right\rangle, \lambda_{b b}=24.0$

FIG. 15: Evolution for polarization correlations along three directions of the open subsystem.

Where there are 8 spins in the bath and the initial state of the subsystem is $1 / \sqrt{2}(|01\rangle+|10\rangle)$. 


\section{B. Concurrence}

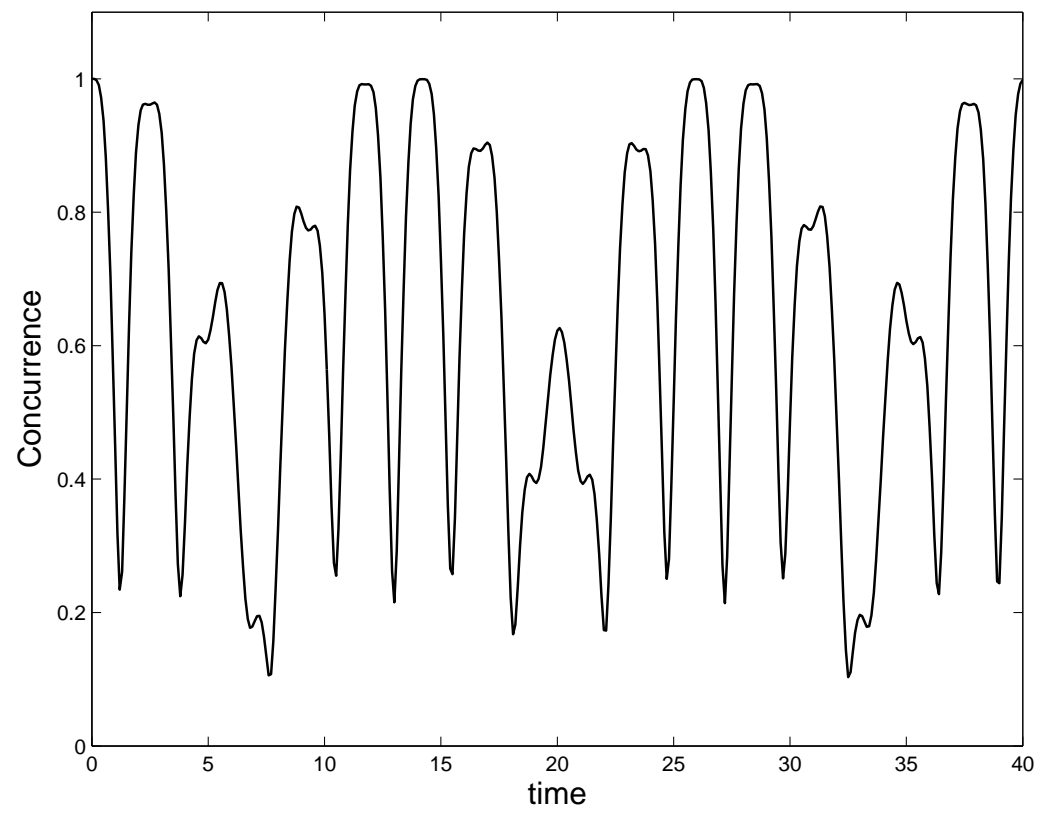

FIG. 16: Evolution of the concurrence of the two subsystem spins for the isolated subsystem. The initial state of the subsystem is $1 / \sqrt{2}(|00\rangle+|11\rangle)$.

The effect of coupling strengths of bath spins on the concurrence are also studied. In figures 16, 17 and 18 we plot the time evolution of the concurrence of three Bell states for the isolated subsystem. An example that the subsystem-bath coupling is considered but the intra-bath coupling strength is zero is showed by figure 19, which can be compared with figure 20] because of the same compaction condition except $\lambda_{b b}$.

Figures 20, 21 and 22 are plots of the evolution of concurrence of the three Bell states of the open subsystem. The number of bath spins is $m=6$ and two coupling strengths of bath spins, $\lambda_{b b}=6.0$ and $\lambda_{b b}=10.0$, are used in the calculation. By comparing the results of $\lambda_{b b}=6.0$ with that of $\lambda_{b b}=10.0$, there are visible improvements of the concurrence by increasing the coupling strength of the bath spins. However, it is found that the influence of the same coupling strengths of bath spins has less effect on the concurrence than on the polarization correlations. From the figure we see that even for the strength $\lambda_{b b}=10.0$, the concurrences are still somewhat different from the isolated cases. It is also noted that the influence is different for different initial stats, it can be seen from figure 20 that for the 


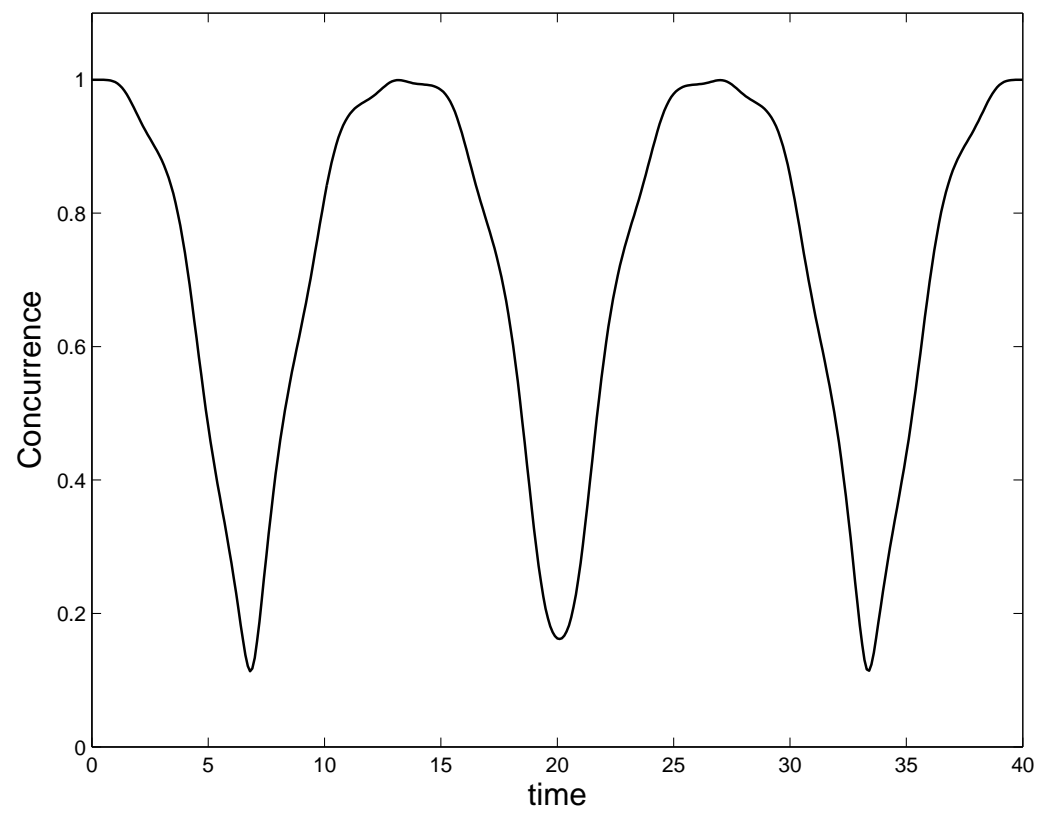

FIG. 17: Evolution of the concurrence of the two subsystem spins for the isolated subsystem. The initial state of the subsystem is $1 / \sqrt{2}(|01\rangle+|10\rangle)$.

case of Bell state $\left|\psi_{S}(0)\right\rangle^{2}=1 / \sqrt{2}(|01\rangle+|10\rangle)$, much better suppression of decoherence is observed than the other two states. Thus we provided the results for a larger bath with the initial state $\left|\psi_{S}(0)\right\rangle^{2}$ in figure 23, where there are $m=8$ bath spins. We found as $\lambda_{b b}=24$, the evolution of concurrence approaches the dynamics of subsystem evolving in isolation. 


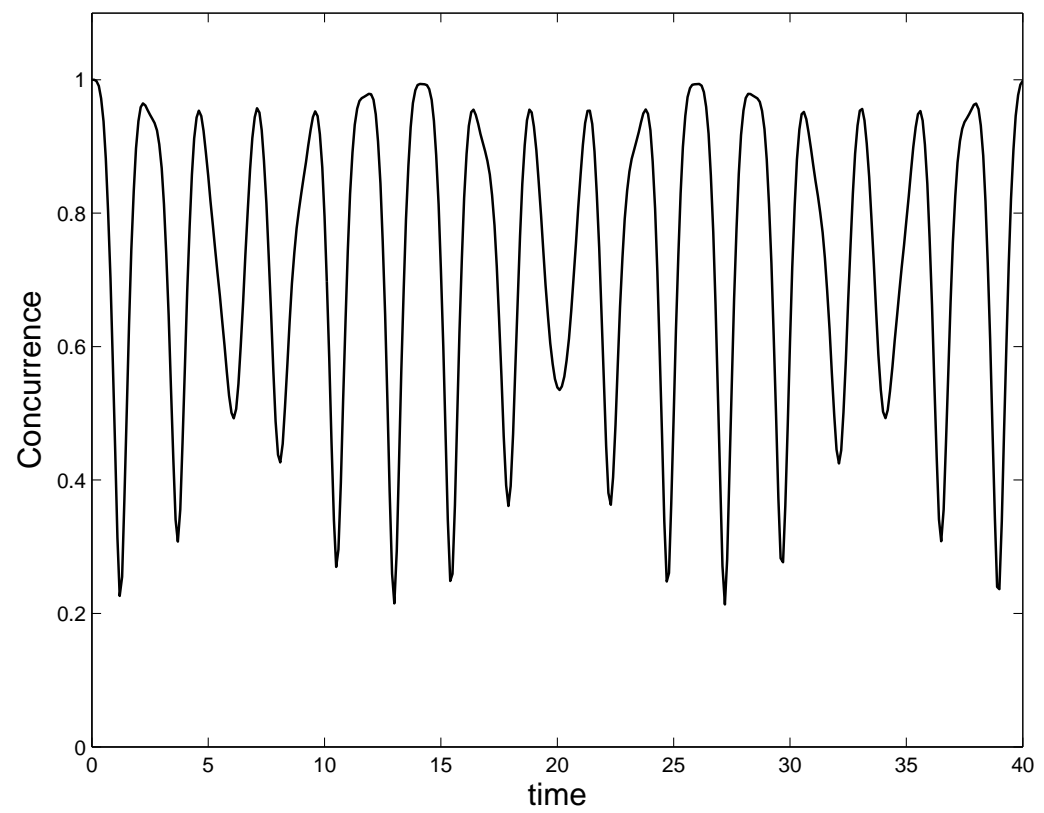

FIG. 18: Evolution of the concurrence of the two subsystem spins for the isolated subsystem. The initial state of the subsystem is $1 / \sqrt{2}(|00\rangle-|11\rangle)$.

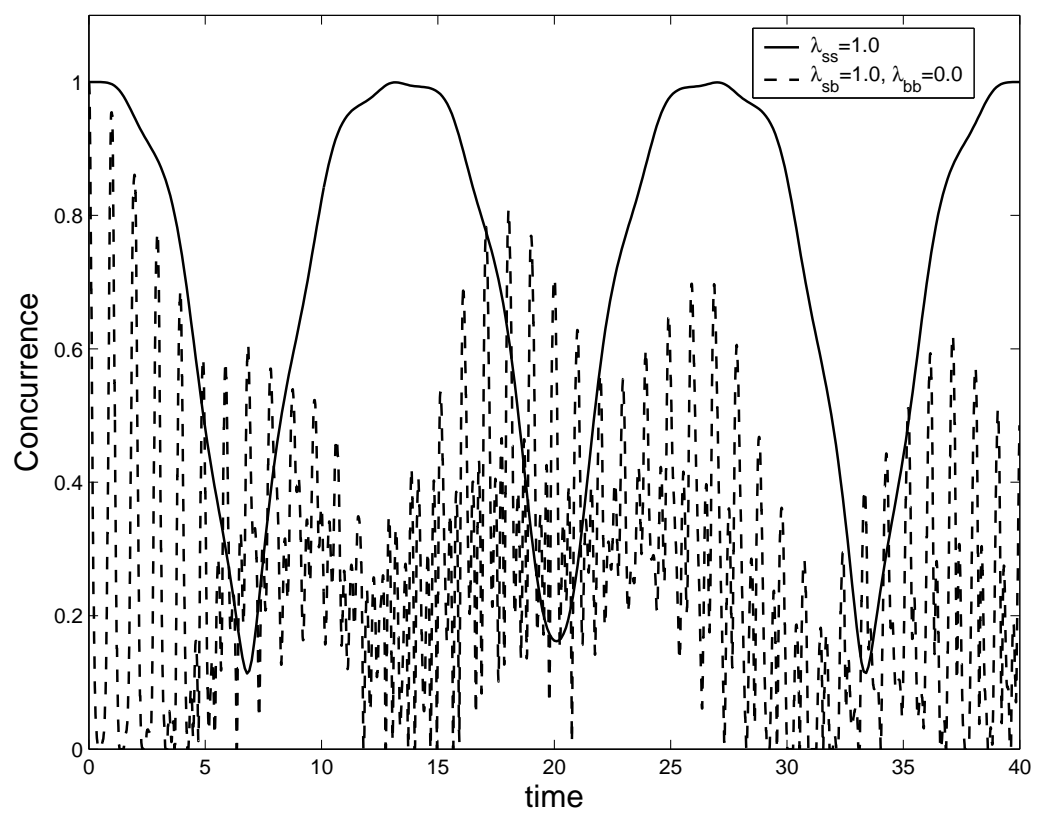

FIG. 19: The evolution of concurrence of the open subsystem. Where there are 6 spins in the bath and the initial state of the subsystem is $1 / \sqrt{2}(|10\rangle+|01\rangle)$ and $\lambda_{s s}=\lambda_{s b}=1.0, \lambda_{b b}=0.0$. 


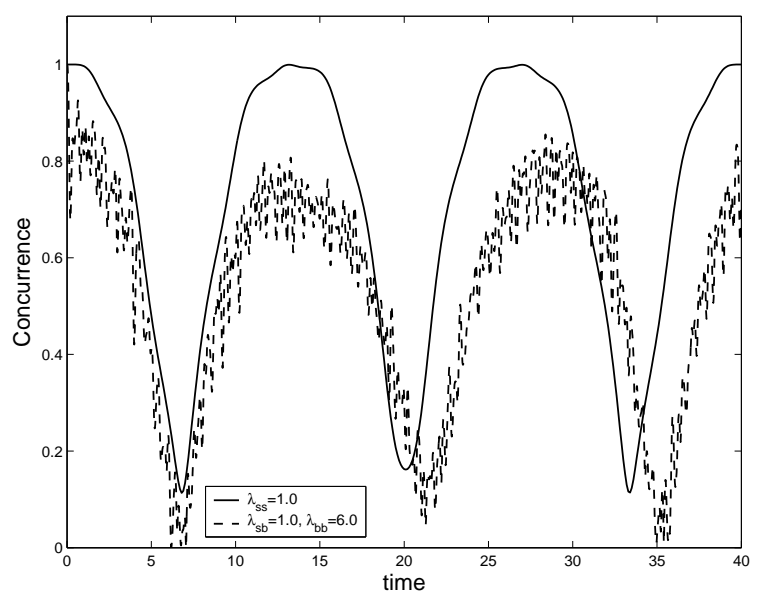

(a) $\lambda_{b b}=6.0$

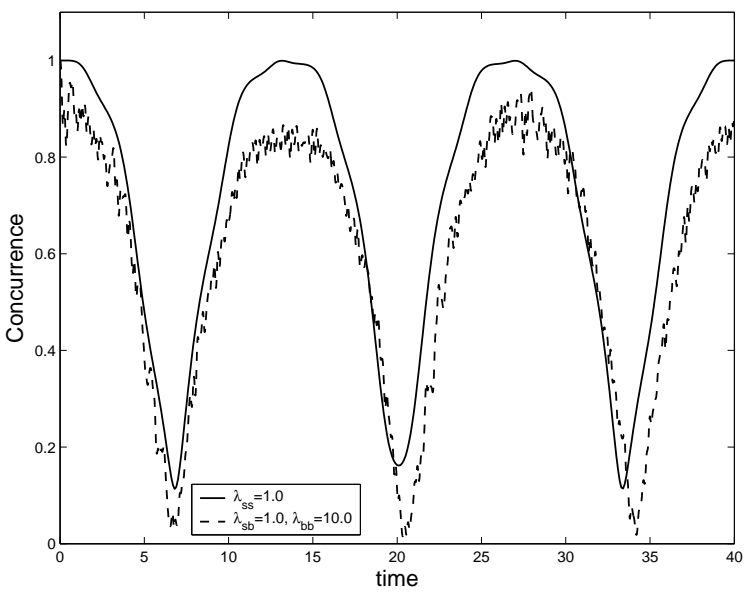

(b) $\lambda_{b b}=10.0$

FIG. 20: The evolution of concurrence of the open subsystem. Where there are 6 spins in the bath and the the initial state of the subsystem is $1 / \sqrt{2}(|01\rangle+|10\rangle)$ and $\lambda_{s s}=\lambda_{s b}=1.0$

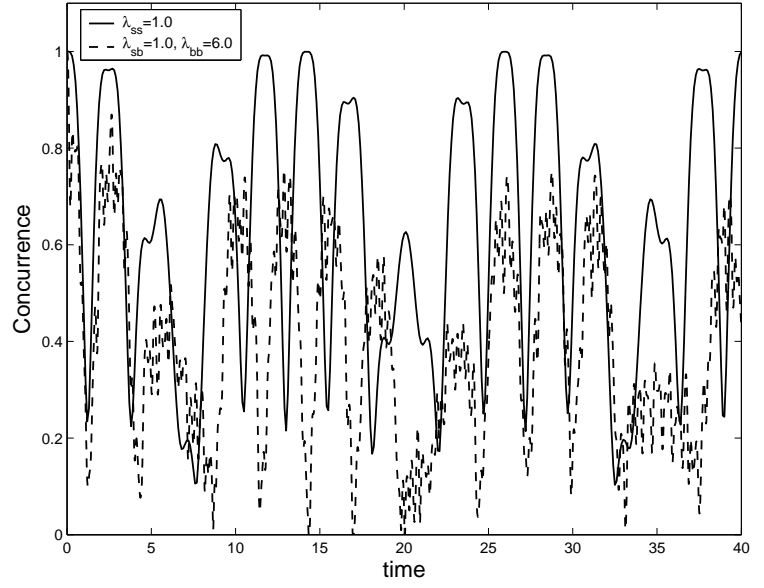

(a) $\lambda_{b b}=6.0$

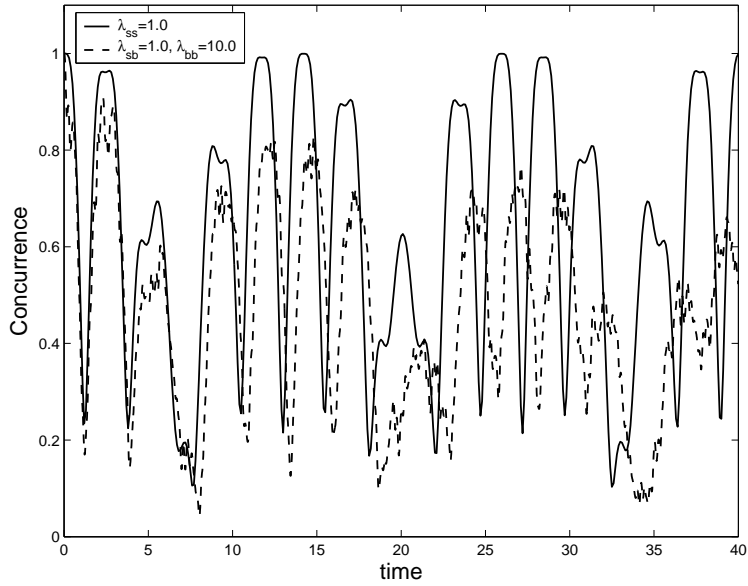

(b) $\lambda_{b b}=10.0$

FIG. 21: The evolution of concurrence of the open subsystem. Where there are 6 spins in the bath and the initial state of the subsystem is $1 / \sqrt{2}(|00\rangle+|11\rangle)$ and $\lambda_{s s}=\lambda_{s b}=1.0$ 


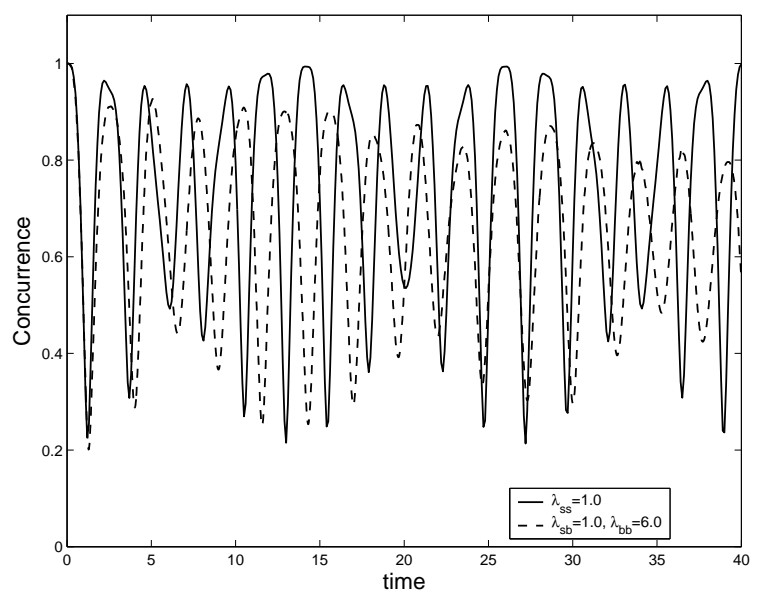

(a) $\lambda_{b b}=6.0$

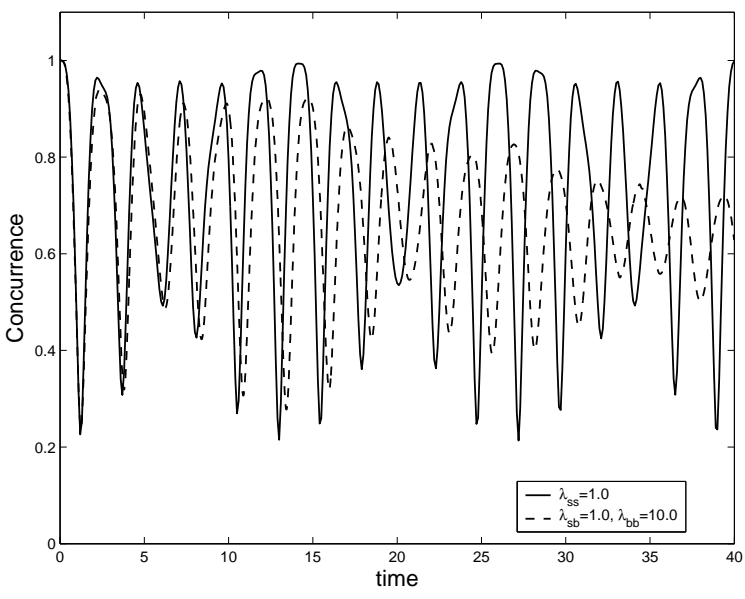

(b) $\lambda_{b b}=10.0$

FIG. 22: The evolution of concurrence of the open subsystem. Where there are 6 spins in the bath and the initial state of the subsystem is $1 / \sqrt{2}(|00\rangle-|11\rangle)$ and $\lambda_{s s}=\lambda_{s b}=1.0$

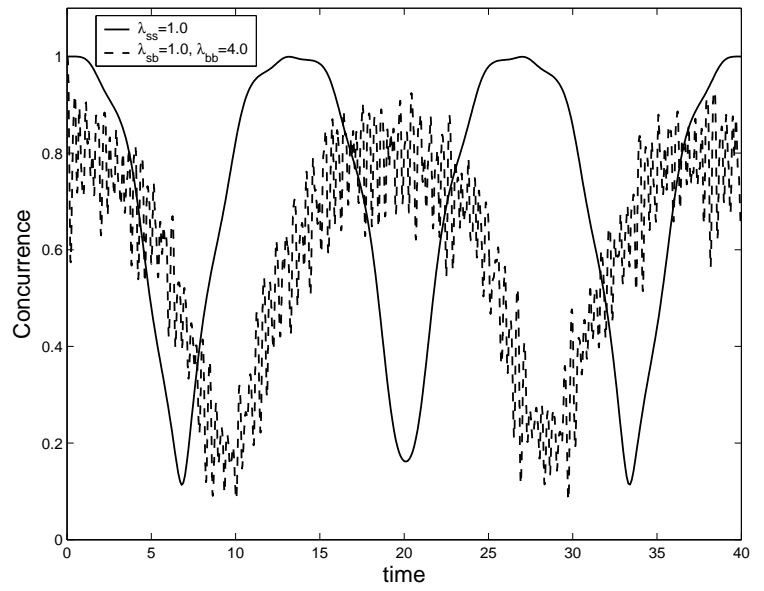

(a) $\lambda_{b b}=4.0$

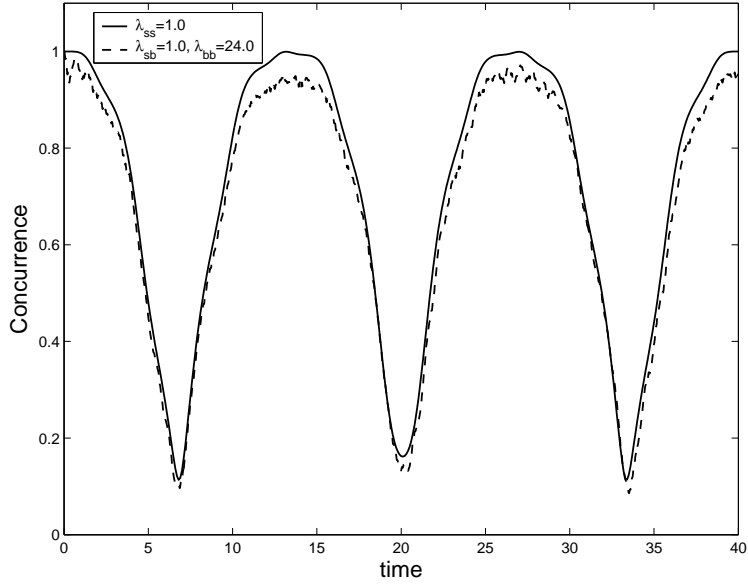

(b) $\lambda_{b b}=24.0$

FIG. 23: The evolution of concurrence of the open subsystem. Where there are 8 spins in the bath and the initial state of the subsystem is $1 / \sqrt{2}(|01\rangle+|10\rangle)$ and $\lambda_{s s}=\lambda_{s b}=1.0$ 


\section{Discussion}

All the results of comparison in the above suggest that if we did not consider the intrabath coupling, or we cannot control the intra-bath coupling, the decoherence occurring in our subsystem due to the bath is very severe. Although the numbers of bath spins in our simulation is not very big, but these bath spins can be regarded as the nearest-neighbors to our open subsystem, the interaction between them $\lambda_{s b}$ is much larger than the coupling exists between subsystem spin and other degree of freedom in the real-world environment. So the bath we considered in this study could form a safeguard device of the open subsystem, which intra-coupling could be adjusted to a high level to counteract the dissipation by itself and the real-world environment.

To understand the physics behind the decoherence suppression effects due to the strong coupling among bath spins, we investigated the states of the bath when the coupling strength changed. The bath spins coupled antiferromagneticly with each other in the $x$ direction, which cause the bath spins in a kind of frustrated state. The other terms in the Hamiltonian favors a aligned ordered state. The final state is the competition between the different terms as well as the thermal fluctuations and turns out to be very complex. However, we believe that the decoherence suppression effects are somehow related to the state ordering of the bath spins.

At temperatures not high enough, the properties of the bath are determined by the few lowest energy levels, so we will concentrate on the lowest energy levels and try to figure out the characteristics of the states. In our representation where the $z$ component of the spin is diagonal, it is hard to see the ordering properties of the states. Since the coupling is in the $x$ direction, we thus transform our states to the $x$ component diagonal representation for clarity. This is simply achieved by the following recipe to each spin state,

$$
\left|\varphi^{i}\right\rangle_{x}=U^{-1}\left|\varphi^{i}\right\rangle_{z}
$$

Where $U$ is a $2 \times 2$ matrix defined as

$$
U=\frac{1}{\sqrt{2}}\left(\begin{array}{cc}
1 & 1 \\
1 & -1
\end{array}\right)
$$


Now we consider a system with 6 bath spins and two spins in the subsystem, the bath states can be expanded with the product state of the form $\left|i_{1} i_{2} i_{3} i_{4} i_{5} i_{6}\right\rangle_{x}=$ $\left|i_{1}\right\rangle_{x}\left|i_{2}\right\rangle_{x}\left|i_{3}\right\rangle_{x}\left|i_{4}\right\rangle_{x}\left|i_{5}\right\rangle_{x}\left|i_{6}\right\rangle_{x}$, here $i_{\alpha}$ equals 0 or 1 , the subscript $x$ means the $x$-component diagonal representation, and $\alpha=1, \cdots, 6$ are the index of the 6 bath spins. There are $2^{6}=64$ product states, and for each product state, the subsystem can be in four states $|00\rangle_{x},|10\rangle_{x},|01\rangle_{x}$ and $|11\rangle_{x}$. Since the Hamiltonian $H_{B}$ is invariant under permutations of the bath spins, so that the states with the same number of "up" spins in the $x$ direction has the same expectation values of energy, thus we can group the states with the same number of "up" spins together. And using the number of "up" spins $n$ to represent such states and denote it as $\| n\rangle$.

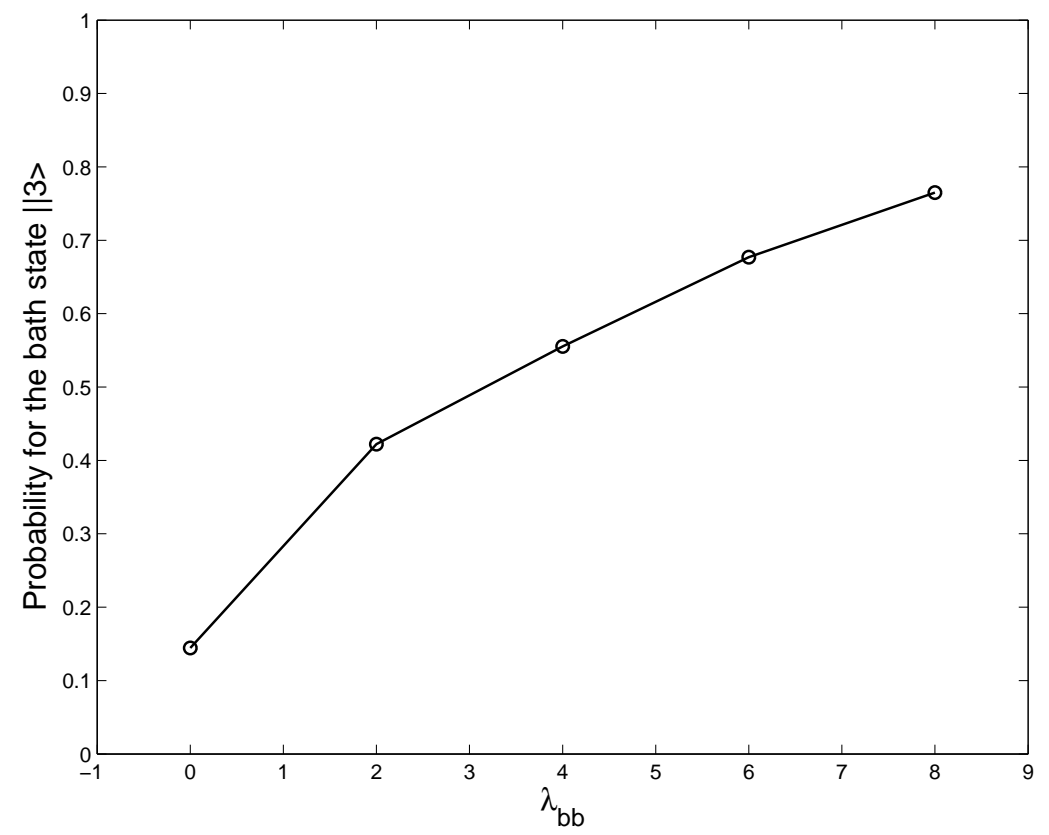

FIG. 24: The probability that the bath state has half of the spins in the "up" state and half in the "down" state as function of the coupling strength $\lambda_{b b}$, the total number of bath states is $m=6$. The initial subsystem state is $1 / \sqrt{2}(|01\rangle+|10\rangle)$ and temperature $T=0.1$.

The probability that each kind of the product states appear in the bath state can be determined from the full density matrix $\rho(t)$, i.e.

$$
P(n)=\operatorname{Tr}_{S}\langle n \| \rho(t)|| n\rangle=\operatorname{Tr}_{s} \sum \omega_{m}\left|\left\langle n \| \Psi_{m}(t)\right\rangle\right|^{2}
$$

The $\mathrm{Tr}_{s}$ means to trace out the subsystem degrees of freedom. In the case of 6 bath spins, we calculated the probabilities for each state $\| n\rangle$ and found that the state $\| 3\rangle$, which has 3 spins 
"up" and 3 spins "down" in the $x$ direction, has the majority probability, and the probability increases with the coupling strength. Figure 24 is the plot of the probability $P(3)$. We see that for small coupling strength, the probability is about 0.1, and as the coupling strength increases, the probability increases monotonically and reaches 0.8 at $\lambda_{b b}=10$, where the big suppression effects was obtained. Based on this observation we conclude that as the coupling strength increases, the bath spins are self organized to a resonant antiferromagnetic ordered state in $x$ direction, one half of the spins are in the "up" state and the other half are in the "down" state. It is this ordering that brings the subsystem to the more coherent state. This mechanism may play an important role in the controlling of the subsystem coherence of quantum device, despite the inevitable influences of thermal noise upon the quantum device, we may also couple the quantum device to a system as described in this paper. By changing the coupling strength of the added bath spins, one may reduce the decoherence to a level for practical applications.

\section{CONCLUSION}

In this paper, we extended the one-center-spin-spin-bath model [1, 14] to a 2-center-spinspin-bath model, which could be thought as an analog to two correlated qubits in quantum computer. By calculating the polarization correlation and concurrence of the subsystem, we found that the spin-bath can play a revival role in the evolution of polarization correlation and entanglement between two subsystem spins. In the process of calculation, we combine the techniques of Ref. [1] and Ref. [26] to reduce the computer resources greatly. The

physics of this suppression was found to be the effect of the antiferromagnetic ordering of the bath spins in $x$ direction. We suggest that the results may be of use in the controlling of decoherence of quantum devices.

This work is supported by the National Nature Science Foundation of China under grant \#10334020 and \#90103035.

[1] Tessieri L and Wilkie J, 2003 J. Phys. A 36 12305-12327

[2] Zurek W H, 1981 Phys. Rev. D 241516 
[3] Zurek W H, 1991 Phys. Today 44(10) 36

[4] Joos E and Zeh H D, et al 2003 Decoherence and the Appearance of a Classical World in Quantum Theory Springer-Verlag

[5] Zurek W H, 2003 Rev. Mod. Phys 75715

[6] Leggett A J, Chakravarty S, Dorsey A T, Fisher M P, Garg A and Zwerger W, 1987 Rev. Mod. Phys. 591

[7] Breuer H P and Petruccione F 2002 The Theory of Open Quantum Systems (Oxford: Oxford University Press)

[8] Nielson M A and Chuang I L 2000 Quantum Computation and Quantum Information (Cambridge, England: Cambridge University Press)

[9] Caldeira A O and Leggett A, 1983 J Ann. Phys., NY 149, 374

[10] Prokofev N V and Stamp P C, 2000 Rep. Prog. Phys. 63669

[11] Gardiner C W, 1991 Quantum Noise (Berlin, Heidelberg, New York: Springer-Verlag)

[12] Shresta S, Anastopoulos C, Dragulescu A and Hu B L, 2004 arXiv:quant-ph/0408084 v1 13

[13] Estreicher S K, Weber J, Derecskei-Kovacs A and Marynick D S, 1997 Phys. Rev. B 555037

[14] Dawson C M, Hines A P, Mekenzie R H and Milburn G J, 2005 Phys. Rev. A 71052321

[15] Xiang S H and Song K H, 2006 Acta Physica Sinica 55(2) 529

[16] Liu T K, Wang J S, Feng J and Zhan M S, 2005 Chinese Physics 14(3) 536

[17] Shan C J and Xia Y J, 2006 Acta Physica Sinica 55(4) 1585

[18] Ekert A and Jozsa R, 1996 Rev. Mod. Phys. 68733

[19] Bennett C H, Bernstein H J, Popescu S and Schumacher B, 1996 Phys. Rev. A 532046

[20] Hill S and Wootters W K, 1997 Phys. Rev. Lett. 785022

[21] Wootters W K, 1998 Phys. Rev. Lett. 802245

[22] Davies G, 1981 Rep. Prog. Phys. 44787

[23] Bell J S, 1987 Speakable and unspeakable in Quantum Mechanics (Cambridge: Cambridge University Press)

[24] Dobrovitski V V and De Raedt H A, 2003 Phys. Rev. E 67056702

[25] Hu X G, 1999 Phys. Rev. E 592471

[26] Jing J and Ma H R, 2006 Phys. Rev. E accepted

[27] G. Arfken 1985 Mathematical Methods of Physicists 3rd ed (New York: Academic)

[28] Shi M J, Du J F and Zhu D P, 2000 Acta Physica Sinica 49(5) 825 